\title{
The Political Economy of Entry Barriers*
}

\author{
Toshihiko Mukoyama \\ Federal Reserve Board \\ and \\ University of Virginia \\ tm5hs@virginia.edu
}

\author{
Latchezar Popov \\ University of Virginia \\ lap4d@virginia.edu
}

November 2012

\begin{abstract}
We study a political economy model of entry barriers. Each period the policymaker determines whether to impose a high barrier on entry, and the special interest groups try to influence the policymaker's decision. Entry is accompanied by creative destructionwhen many new firms enter, old firms are more likely to be driven out of the market. Therefore the current incumbents (industry leaders) tend to lobby for a higher entry barrier and potential entrants (industry followers) are likely to lobby for a freer environment for entry. We analyze both static and dynamic versions of the model to examine which environment supports a policy that blocks entry. In the dynamic version of the model, the economy can exhibit various different dynamics, such as multiple steady states and policy-induced cycles.
\end{abstract}

Keywords: political economy, lobbying, entry and exit JEL Classifications: D72; E60; L51; O25

${ }^{*}$ We thank useful comments from Amanda Kurzendoerfer, B. Ravikumar, and seminar participants as well as conference participants at the APET Workshop on the Political Economy of Development, the Federal Reserve Bank of St. Louis, and the Midwest Economic Theory and International Trade Meetings. All errors are ours. The views expressed in this paper are solely the responsibility of the authors' and should not be interpreted as reflecting views of the Board of Governors of the Federal Reserve System or of any other person associated with the Federal Reserve System. 


\section{Introduction}

Many recent studies show that reallocation of productive resources are at the heart of growth process. For example, using the Census of Manufactures data, Foster, Haltiwanger, and Krizan (2001, Table 8.4) attribute about half of the multifactor productivity growth in the U.S. manufacturing sector during 1977-1987 to the reallocation of production resources across plants. In particular, $26 \%$ of productivity growth is due to the entry and exit of plants during this 10 year period.

The process of entry and exit, which is one part of the reallocation process, has received substantial attention in the context of economic growth during recent decades. The process often called "creative destruction" has been strongly associated with the entry and exit of firms - new firms enter the market with new products and technologies, driving out old inefficient firms. ${ }^{1}$

While many economists agree that creative destruction is essential in the growth process, many countries institute policies that act as impediments to entry. In an influential work, de Soto $(1989,2000)$ documents that starting up a new firm in Peru requires many legal procedures and substantial monetary cost. Later, Djankov et al (2002) describe in detail how entry regulations (taking many forms) differ across countries. ${ }^{2}$ These policies, serving as entry barriers, can be a major obstacle to reallocation and productivity growth. ${ }^{3}$ In fact, recent studies such as Barseghyan and DiCecio (2011) and Moscoso Boedo and Mukoyama (2012) suggest that high entry costs contribute significantly to lowering productivity in poor countries.

A natural question is, why do some countries institute such a policy while others do not? Why do they impose inefficiency on themselves by imposing obstacles to the process of creative destruction? Under what kind of environment do countries conduct such policies?

\footnotetext{
${ }^{1}$ Foster, Haltiwanger, and Krizan (2006) find that almost all the labor productivity growth in the U.S. retail trade sector in the 1990s is accounted for by more productive entering establishments replacing less productive exiting establishments.

${ }^{2}$ The World Bank provides more recent updates (see http://www.doingbusiness.org/).

${ }^{3}$ Many recent empirical papers show that these regulations indeed reduce entry. See Djankov (2009) for a survey.
} 
Why do these policies persist for an extended period of time? These are the questions that motivate our study.

In this paper, we build a simple political economy model of policy choice in an economy that is characterized by creative destruction. In building our model, we draw our inspiration from Olson (1982). Analyzing the behavior of special interest groups, he argues that "It would be in the interest of these groups that are organized to increase their own gains... This would include choosing policies that, though inefficient for the society as a whole, were advantageous for the organized groups because the costs of the policies fell disproportionately on the unorganized" (p. 37). As a result, a socially inefficient policy can be chosen due to the influence of the special interest groups. We model the determination of the policy on firm entry regulations as a special case of his analysis. In our model, there are two special interest groups, or "coalitions," who typically have opposite policy preferences. One is the group of incumbent firms who do not want to be driven out of the market by new entrants, and thus favor a policy that makes entry difficult. The other is the group of the potential entrant firms who want a policy that allows them to enter easily. We do not model consumers - they are "the unorganized" who suffer from the cost of inefficient production or lack of new products when a policy that is detrimental to new entry is selected.

A recent work by Acemoglu and Robinson (2012) expresses a related view. They argue that a society which is ruled by "a narrow elite" who pursues their own benefit at the expense of the mass of the people tend to stagnate. They call such an organization ("rules") of society "extractive institutions" and contrast them with "inclusive institutions" which allow the participation of the mass of the people in economic activities. Permitting entry of new businesses and creative destruction is a part of inclusive institutions - under extractive institutions, innovative new technologies are often blocked due to political opposition. They write "The fact that they [the elites] have much to lose from creative destruction means not only that they will not be the ones introducing new innovations but also that they will often resist and try to stop such innovations" (pp. 183-184). Furthermore, similarly to our own view, they emphasize the role of organization within groups: "It is also necessary to 
consider more broadly the factors that determine how political power is distributed in society, particularly the ability of different groups to act collectively to pursue their objectives or to stop other people from pursuing theirs" (pp. 42-43).

Our interest in the entry policy is motivated by two strands of literature. One is the aforementioned recent literature that focuses on the effect of entry costs in accounting for international income differences. The other is the endogenous growth literature which features creative destruction. In the Schumpeterian models of economic growth, such as Grossman and Helpman (1991) and Aghion and Howitt (1992), the engine of growth is the entry of firms with better quality products. When new firms enter, old firms with lower-quality products are driven out of the market. Restricting entry in these models results in slower growth and (typically) a large welfare loss. In this paper we focus on the positive analysis of policy determination and therefore we do not make explicit welfare statements. ${ }^{4}$ However, a background assumption is that a policy choice that is detrimental to creative destruction can be quite costly. $^{5}$

We build on the framework of the menu auction by Bernheim and Whinston (1986) in modeling the policy determination in an environment with special interest groups. In characterizing our model, we utilize Bergemann and Välimäki's (2003) dynamic extension of Bernheim and Whinston (1986). The menu auction framework is widely used in the context of international trade policy — for example, in Grossman and Helpman (1994), special interest groups lobby for trade policies. In this paper, we start from a static model that is a direct application of the Bernheim and Whinston (1986) framework, and then contrast the results with the dynamic model.

There are some other recent studies that are influenced by Olson's work. Krusell and Ríos-Rull (1996) consider a model where agents vote for the adoption of new technology.

\footnotetext{
${ }^{4}$ In Appendix A, we present a version of our model with general equilibrium and endogenous growth. There we show that inhibiting creative destruction results in some welfare costs.

${ }^{5}$ Parente and Prescott (1999) share our view that the entry of new firms with new technology is essential in growth and development and thus barriers to entry can cause inefficiencies. They consider a setting where there is a coalition of (incumbent) factor suppliers associated with inferior technology and the new entrants have to make a costly investment to overcome the coalition's resistance. In contrast to our political economy model, in Parente and Prescott (1999) there are no explicit political processes that affect the ease of entry.
} 
While young agents like new technology, older agents who have a vested interest in old technology may want to block the adoption of new technology. There are two differences between their model and ours. First, they employ an overlapping-generations model and the generational conflict plays a large role in their mechanism, while in our model the conflict is among firms who live for an infinite horizon. Second and more importantly, they employ majority voting as the political process, while we consider lobbying by special interest groups. This allows us to highlight the role of both the "extensive margin" (how large each special interest group is) and the "intensive margin" (how strongly each firm feels about political options). Since our paper formalizes Olson's (1982) analysis of special interest groups, the inclusion of the intensive margin is of critical importance. It also turns out that our model is substantially simpler to characterize than Krusell and Ríos-Rull's (1996), enabling us to analyze the importance of various parameters in generating different political outcomes.

More recent papers by Bridgman et al (2004) and Bellettini and Ottaviano (2005) are closely related to ours. Both papers consider a model where the special interest groups lobby to influence the adoption of new technology. They both employ an overlapping-generation model, where the "old" do not want a new technology and the "young" do. In Bridgman et al (2004), the old lobby for blocking the new technology, while the young do not participate in lobbying. There is a trade-off in policymaking because the policymaker cares both about the value of GDP and the monetary receipt from the old. This trade-off is characterized by the weight (given as an exogenous parameter) that the policymaker assigns to GDP. In Bellettini and Ottaviano (2005), the old lobby for blocking and the young lobby for adopting new technology. In that paper, the important parameter that determines the relative political power is the population growth rate. Our dynamic model describes the lobbying activities by the firms who live perpetually. An important difference here is that in our model the relative size of each coalition changes over time and endogenously. That is, whether there are many incumbent firms in the following period depends on what policy is currently adopted. This type of feedback from the policy to the coalition size is absent in each of the above studies. The fact that the coalition size is endogenous is crucial in formally analyzing Olson's (1982) 
idea. For example, we later describe a situation that is parallel to Olson's analysis of the "British disease." There, the fact that the special interest group "builds up" during the period of free entry is important in leading to the future policy switch towards the blocking of new entry.

In terms of the results, our dynamic model allows for richer possibilities for the time-series policy outcomes than Krusell and Ríos-Rull (1996), Bridgman et al (2004), and Bellettini and Ottaviano (2005). These papers emphasize the possibility of cyclical policy dynamics, which our model also allows. Unlike these models, our model also permits multiple steady states: two economies with the same set of parameter values and different initial number of incumbents may converge to different steady states with different policy choices. This means that two similar economies may end up having very different level of creative destruction in the long run.

The possibility of multiple steady states provides a key insight into the questions we raised earlier, in particular why some inefficient policies persist. In the situations where multiple steady states can arise, history matters for the political and economic outcome in the long run. The initial distribution of political power affects the economy through the policy choice, and the economic outcome feeds into the future distributions of political power. The political process and the economic process can have a positive feedback on each other, and the initial "bias" of the political power can be strengthened over time. In such a situation, a onetime (temporary) intervention to the policy can have a permanent effect on the political and economic outcome. In an influential book, North (1990) emphasizes the interaction between the political process and the economic process, and writes "political rules in place lead to economic rules, but the causality runs both ways. That is, property rights and hence individual contracts are specified and enforced by political decision-making, but the structure of economic interests will also influence the political structure" (p. 48). North emphasizes the "path-dependent" nature of the institutional and economic change, and our multiple steady state result can be viewed as one example of such a path-dependence. Acemoglu and Robinson (2012) also emphasize the "virtuous circle" and "vicious circle" between political 
institutions (corresponding to the distribution of political power in our context) and economic institutions (the resulting economic policy regimes in our context) that gives rise to a very different set of persistent institutions that resulted from seemingly small initial differences.

The paper is organized as follows. In Section 2, we characterize a simple static model. Section 3 presents the dynamic model. We first analyze the general characteristics of the equilibrium outcome, and then illustrate special cases to obtain sharper characterization. Section 4 concludes.

\section{Static model}

In this section we consider a simple static model. There is a continuum of firms with a total population of one. There are two types of firms, leaders and followers. Leaders are the incumbent firms who are already in the market. In the dynamic model of Section 3 , they are the firms who operated in the previous period. We denote the mass of leaders by $L$. The rest of the firms are followers, who are potential entrants outside the market. In the dynamic model, they are the firms who did not operate in the previous period. Their mass is $(1-L)$.

There are two political regimes, which are chosen in the beginning of the period by a mechanism that we describe in detail below. The political regime is either free entry or entry blocking. In the free entry regime, there is a substantial amount of creative destruction in the economy, accompanied by a high exit rate of leaders and a high entry rate of followers. In the entry blocking regime, the amount of creative destruction is limited by a government policy, and the exit rate of leaders and the entry rate of followers are both low. ${ }^{6}$

Formally, let $a \in\{e, b\}$ be the policy regime. Here $e$ represents free entry and $b$ represents entry blocking. At the beginning of the period, each leader is hit by a shock (that is i.i.d. across firms) which determines whether it can stay in the market or not. Under policy regime

\footnotetext{
${ }^{6}$ Although we do not explicitly model how the policy affects entry and exit rates, the policies we have in mind are the government regulations related to firm entry (official procedures and costs for entry), documented earlier in the influential work by de Soto $(1989,2000)$ and later by Djankov et al (2002) and the World Bank (http://www.doingbusiness.org/). These include entry fees, procedures to obtain permits, requirements for government-provided licenses, minimum capital requirements, and other regulations that make it difficult for new firms to enter. In a broad sense, subsidies to the current incumbents and strong patent protections can also be viewed as policies that act as obstacles to entry.
} 
$a$, the probability of a leader staying in the market in the current period is $\left(1-\delta_{a}\right)$. Thus $\delta_{a} \in(0,1)$ is the exit probability. We assume that $\delta_{e}>\delta_{b}$, that is, a leader is more likely to stay in the market in the entry blocking regime. Similarly, at the beginning of the period each follower is hit by a shock that determines whether it can enter the market. The probability of a follower (a potential entrant) actually entering in the market in the current period is $\lambda_{a} \in(0,1)$, where $\lambda_{e}>\lambda_{b}$. A follower is more likely to enter during the free entry regime.

The timing is the following. First, there is a political process (detailed below) that determines the policy regime $a$. Second, firms are hit by a random shock of entry and exit. Since a leader exits the market with probability $\delta_{a}$ and there is a continuum of leaders with mass $L$, the population $\left(1-\delta_{a}\right) L$ of leaders stays in the market and operates. A follower enters the market with probability $\lambda_{a}$, and thus population $\lambda_{a}(1-L)$ of followers enter the market and operate. Third, the firms in the market operate and earn profit. We assume that a leader who operates earns $\pi_{\ell}>0$ amount of profit. A follower who operates earns $\pi_{f}$ amount of profit. (Below, we always use the subscript $\ell$ for leaders and the subscript $f$ for followers.) Leaders who exited and followers who did not enter earn zero profit.

In this paper, we do not make an assumption about whether $\pi_{\ell}$ is larger than $\pi_{f}$. In our view, there are equally compelling arguments that one may be larger than the other. If there is an element of "learning by doing" in the industry, a firm who stayed longer in the industry would tend to have a higher profitability. In this case, it is more likely that $\pi_{\ell}>\pi_{f}$. In contrast, if new entrants can enter the market with the latest technology and equipment, thus having advantage due to "vintage effect," it is more plausible that $\pi_{f}>\pi_{\ell}$. In the following, we will consider both cases.

The political process is modeled as a common agency game. Based on Bernheim and Whinston (1986), we assume that special interest groups bid in a first-price menu auction. In our context, there is a policymaker (auctioneer in Bernheim and Whinston's terminology) who decides the policy regime $a$. The policymaker does not have her own preferences over the two possible regimes. Rather, her decision is influenced by monetary payments of special interest groups (bidders in Bernheim and Whinston's terminology). One can interpret these 
payments as campaign contributions, lobbying, or bribes. In our formulation, the special interest groups first specify the conditional payments for each policy option (they specify the amount of money they pay conditional on the policymaker choosing that particular policy option), and the policymaker chooses the policy that maximizes the sum of these payments. We assume that, instead of acting individually, the leaders form one coalition (special interest group) and the followers form another coalition. Although we do not explicitly model how the coalitions are formed, we believe that this is the most natural way of dividing the firms into groups, given that within each coalition the firms share the same interest. The expected profit for the leader coalition when policy $a$ is chosen is

$$
V_{\ell}(a) \equiv L\left(1-\delta_{a}\right) \pi_{\ell}
$$

and the expected profit for the follower coalition is

$$
V_{f}(a) \equiv(1-L) \lambda_{a} \pi_{f}
$$

Within a coalition, the payments for the auction are shared equally. The objective of an individual firm is to maximize the expected profit minus the political contribution.

The political process is formulated with the following timing. First, each coalition specifies the conditional payments to the policymaker for each policy option. We denote the conditional payments from coalition $i(i=\ell, f: \ell$ for leaders and $f$ for followers $)$ for policy $a$ as $R_{i}(a) \geq 0$. Second, upon observing the conditional payments, the policymaker chooses the policy option $a$ which maximizes $R_{\ell}(a)+R_{f}(a)$. The resulting net gain for coalition $i$ when the policy $a$ is chosen is $N_{i}(a) \equiv V_{i}(a)-R_{i}(a)$.

In order to define the Truthful Nash Equilibrium, we first define a truthful strategy.

Definition $1 R_{i}(a)$ (for $i=\ell, f$ ) is said to be a truthful strategy relative to $a^{*}$ if, for $a=e, b$, either

$$
N_{i}(a)=N_{i}\left(a^{*}\right)
$$

or

$$
N_{i}(a)<N_{i}\left(a^{*}\right) \text { and } R_{i}\left(a^{*}\right)=0 \text {. }
$$


holds.

Now, the definition of the Truthful Nash Equilibrium is as follows.

Definition $2\left(\left\{R_{i}(a)\right\}_{i=\ell, f ; a=e, b}, a^{*}\right)$ is a Nash Equilibrium if $(i) R_{\ell}\left(a^{*}\right)+R_{f}\left(a^{*}\right) \geq R_{\ell}(a)+$ $R_{f}(a)$ for all a and (ii) for each $i$, there is no other strategy that yields a net payoff greater than $N_{i}\left(a^{*}\right)$ given $R_{j}(a)(j \neq i ; a=e, b)$. It is called a Truthful Nash Equilibrium if it is a Nash Equilibrium and each coalition plays a truthful strategy relative to $a^{*}$.

Bernheim and Whinston (1986) show that, in certain settings that contain this section's model as a special case, the Truthful Nash Equilibrium is unique (see their Corollary 1).

It is straightforward to check the following result. (Proof is omitted.)

Proposition 1 The Truthful Nash Equilibrium in the static model is:

- If $V_{\ell}(b)-V_{\ell}(e) \geq V_{f}(e)-V_{f}(b)$,

$$
\begin{gathered}
\left(R_{\ell}(e), R_{\ell}(b)\right)=\left(0, V_{f}(e)-V_{f}(b)\right) \\
\left(R_{f}(e), R_{f}(b)\right)=\left(V_{f}(e)-V_{f}(b), 0\right)
\end{gathered}
$$

and $a^{*}=b$ is selected.

- Otherwise,

$$
\begin{aligned}
& \left(R_{\ell}(e), R_{\ell}(b)\right)=\left(0, V_{\ell}(b)-V_{\ell}(e)\right) \\
& \left(R_{f}(e), R_{f}(b)\right)=\left(V_{\ell}(b)-V_{\ell}(e), 0\right)
\end{aligned}
$$

and $a^{*}=e$ is selected.

The payoff (before the political contributions are made) for the leader coalition is maximized by the regime of entry blocking $b$. The excess payoff for the leader from the policy $b$ is, from (1),

$$
V_{\ell}(b)-V_{\ell}(e)=L\left(\delta_{e}-\delta_{b}\right) \pi_{\ell},
$$


and this is the upper bound of the political contribution that the leader coalition is willing to pay in order to obtain the policy $b$. Similarly, the excess payoff from the policy $e$ for the follower coalition is given by, from (2),

$$
V_{f}(e)-V_{f}(b)=(1-L)\left(\lambda_{e}-\lambda_{b}\right) \pi_{f}
$$

When $L\left(\delta_{e}-\delta_{b}\right) \pi_{\ell} \geq(1-L)\left(\lambda_{e}-\lambda_{b}\right) \pi_{f}$, the leaders can "outbid" the followers. Thus it is easy to see that the outcome policy will be $b$ in any Nash equilibrium in this case. It is an equilibrium strategy for the follower coalition to offer any amount up to $L\left(\delta_{e}-\delta_{b}\right) \pi_{\ell}$ as $R_{f}(e)$ (and the leader coalition has to "match" this amount as $R_{\ell}(b)$ in order to win), since the follower coalition "loses the bid" in equilibrium and does not have to pay $R_{f}(e)$. Thus there are multiple Nash equilibria, with different values of $R_{f}(e)$ and $R_{\ell}(b)$, with the equilibrium policy $b$. But the equilibria with $R_{f}(e)>V_{f}(e)-V_{f}(b)$ are not very reasonable since this bid is similar to an "empty threat" - they are willing to offer it because they know that they will not pay it. This is the reason we need the refinement of Truthful Nash Equilibrium (which is unique here).

Using (3) and (4), the condition that $a^{*}=b$ is selected can be rewritten as:

$$
\frac{L}{(1-L)} \frac{\left(\delta_{e}-\delta_{b}\right) \pi_{\ell}}{\left(\lambda_{e}-\lambda_{b}\right) \pi_{f}} \geq 1 .
$$

The term $L /(1-L)$ represents the "extensive margin" (the size of the coalitions). The term $\left(\delta_{e}-\delta_{b}\right) \pi_{\ell} /\left(\lambda_{e}-\lambda_{b}\right) \pi_{f}$ represents the "intensive margin." Each leader firm's net gain (in expected value) when policy $b$ is chosen is $\left(\delta_{e}-\delta_{b}\right) \pi_{\ell}$ and each follower firm's net loss (in expected value) when policy $b$ is chosen is $\left(\lambda_{e}-\lambda_{b}\right) \pi_{f}$. This condition shows that both margins matter.

The condition (5) can also be rewritten as a threshold rule:

$$
a= \begin{cases}e & \text { if } L<L^{*} \\ \{e, b\} & \text { if } L=L^{*} \\ b & \text { if } L>L^{*}\end{cases}
$$

where the threshold $L^{*}$ is

$$
L^{*}=\frac{\left(\lambda_{e}-\lambda_{b}\right) \pi_{f}}{\left(\lambda_{e}-\lambda_{b}\right) \pi_{f}+\left(\delta_{e}-\delta_{b}\right) \pi_{\ell}} .
$$


If the mass of leaders $L$ is sufficiently large, the entry blocking policy is selected. Here $L$ is given exogenously, but in reality $L$ changes over time. In particular, the current value of $L$ is determined as a result of past entry and exit. This means that the past policies affect the current $L$, which in turn affects the current policy. One can easily see that there is a non-trivial dynamic interaction between the policy choice and the population of the leaders. We examine this interaction in the next section by extending the model to a dynamic setup.

Our equilibrium formulation allows us to characterize the final payoff for each agent. Define the net payoff for each firm in coalition $i(i=\ell, f)$ as

$$
n_{\ell} \equiv \frac{\left.N_{\ell}\left(a^{*}\right)\right)}{L}
$$

and

$$
n_{f} \equiv \frac{\left.N_{f}\left(a^{*}\right)\right)}{1-L}
$$

It is straightforward to show that

$$
n_{\ell}= \begin{cases}\left(1-\delta_{e}\right) \pi_{\ell} & \text { if } L<L^{*} \\ \frac{L^{*}}{L}\left(1-\delta_{e}\right) \pi_{\ell}+\left(1-\frac{L^{*}}{L}\right)\left(\left(1-\delta_{b}\right) \pi_{\ell}+\left(\lambda_{e}-\lambda_{b}\right) \pi_{f}\right) & \text { if } L \geq L^{*}\end{cases}
$$

and

$$
n_{f}= \begin{cases}\frac{1-L^{*}}{1-L} \lambda_{b} \pi_{f}+\left(1-\frac{1-L^{*}}{1-L}\right)\left(\lambda_{e} \pi_{f}+\left(\delta_{b}-\delta_{e}\right) \pi_{\ell}\right) & \text { if } L<L^{*} \\ \lambda_{b} \pi_{f} & \text { if } L \geq L^{*}\end{cases}
$$

From these, we can see that $n_{\ell}$ is constant in $L$ when $L<L^{*}$ and increasing in $L$ when $L \geq L^{*}$. We can also see that $n_{f}$ is decreasing in $L$ when $L<L^{*}$ and constant in $L$ if $L \geq L^{*}$. The revenue of the policymaker is

$$
R_{\ell}\left(a^{*}\right)+R_{f}\left(a^{*}\right)= \begin{cases}L\left(\delta_{b}-\delta_{e}\right) \pi_{\ell} & \text { if } L<L^{*} \\ (1-L)\left(\lambda_{e}-\lambda_{b}\right) \pi_{f} & \text { if } L \geq L^{*}\end{cases}
$$

Thus the revenue is increasing in $L$ up to $L^{*}$ and decreasing in $L$ after $L^{*}$. That is, the government revenue is maximized when $L=L^{*}$. The policymaker can extract more revenue from private agents when they have "neck-and-neck" political power. An implication of our model is that when the policymaker is revenue-maximizing, she prefers a situation in which two private coalitions have close political power, rather than where one of them strongly dominates the other. In this sense, the policymaker has a "preference for conflict among private coalitions." 


\section{Dynamic model}

In this section, we extend the model in the previous section to a dynamic one. It turns out that our model permits a variety of equilibrium dynamics, depending on parameter values. In particular, we illustrate that there are situations that resemble the "British disease," an equilibrium with multiple steady states, and an outcome exhibiting cyclical dynamics.

\subsection{Setup}

Time is discrete with infinite horizon. Each firm lives infinitely and maximizes the present value of the sum of the expected profit minus the political payment, discounted by the discount factor $\beta \in(0,1)$. The policymaker also lives infinitely, and discounts the future at the same rate as the firms.

The events during a period follow the same order as in the static model. At the start of period $t$, each coalition (the leader coalition or the follower coalition, details are discussed later) announces the schedule of conditional payments for the current period. Then the policymaker decides on the policy $a_{t}$. After entry and exit occurs, each firm that is in the market earns profit.

We call a firm a "leader" if it operated in the market at period $t-1$ and a "follower" if it did not operate at period $t-1$. The mass of the leaders at the start of time $t$ (before the time $t$ entry and exit occur) is denoted by $L_{t}$. Again, the probability that a follower enters is $\lambda_{a}$ and the probability that a leader exits is $\delta_{a}$ when the current policy is $a$. If a leader firm stays in the market, it earns $\pi_{\ell}$ amount of profit, and if a follower firms enters, it earns $\pi_{f}$. Firms outside the market earn zero. As we will explain in detail later, we assume that both the firms and the policymaker cannot commit to their future behavior.

There are three important aspects that make the dynamic model different from the static model. First, the population of the leader, $L_{t}$, changes over time. Moreover, its future values are influenced by the current policy. Since the value of $L_{t}$ influences the policy decision at time $t$, there is a "propagation" of current policy to the future policy through the movement of $L_{t}$. One particular point of interest here is how the policy is shaped in the long run, as $L_{t}$ 
evolves endogenously over time. Second, since a leader can become a follower (and a follower can become a leader) in the future with some probability, and the transition probabilities are affected by the political decision, the policy has an effect on the "planning horizon" of the forward-looking firms. Third, since the policymaker is also forward-looking, it may become easier or harder for the firms to influence the policymaker compared to the static setting.

Since there is a continuum of firms, the transition of $L_{t}$ follows a deterministic law of motion

$$
L_{t+1}=\mathcal{L}\left(a, L_{t}\right)
$$

when $a_{t}=a$. Here, $\mathcal{L}\left(a, L_{t}\right)$ denotes the value of $L$ at period $t+1$ when the period- $t$ value of $L$ is $L_{t}$ and the period-t policy is $a \in\{e, b\}$. Under our assumption of entry and exit (and abusing the law of large numbers as is common in the models with continuum of agents), $\mathcal{L}\left(a, L_{t}\right)$ is a linear function of $L_{t}$ for a given choice of $a$ :

$$
L_{t+1}=\lambda_{a}\left(1-L_{t}\right)+\left(1-\delta_{a}\right) L_{t} .
$$

This can be rewritten as

$$
L_{t+1}=\bar{L}_{a}+\left(L_{t}-\bar{L}_{a}\right)\left(1-\lambda_{a}-\delta_{a}\right)=\bar{L}_{a}+\left(L_{t}-\bar{L}_{a}\right) \gamma_{a}
$$

where $\bar{L}_{a} \equiv \lambda_{a} /\left(\lambda_{a}+\delta_{a}\right)$ is the steady state with policy $a$ - that is, the value of $L$ that is unchanged as long as policy $a$ is chosen and $\gamma_{a} \equiv 1-\lambda_{a}-\delta_{a}$ is the persistence of the deviation from the steady state. When $\left|\gamma_{a}\right|$ is small, $L_{t}$ approaches $\bar{L}_{a}$ quickly during the period in which $a$ is chosen.

In the following, we assume that $\gamma_{e}=1-\lambda_{e}-\delta_{e} \geq 0$. This automatically implies that $\gamma_{b}=1-\lambda_{b}-\delta_{b}>0$ since $\lambda_{e}>\lambda_{b}$ and $\delta_{e}>\delta_{b}$. This restriction rules out "mechanical" cyclical dynamics of $L_{t}$ with the same policy: for example, when $1-\lambda_{e}-\delta_{e}<0$ and $L_{t}<\bar{L}_{e}$, $L_{t+1}>\bar{L}_{e}$ holds when $a_{t}=e$. If $a_{t+1}=e$ in addition, $L_{t+2}<\bar{L}_{e}$ holds and so on- even when the policy choice is constant, the value of $L_{t}$ oscillates. We do not think this cyclical behavior is interesting (as opposed to the "genuine" cyclical behavior we characterize later in Section 3.4.3), since this is mechanically driven by the size of $\lambda_{a}$ and $\delta_{a}$ (this dynamics appears when 
$\lambda_{a}$ and $\delta_{a}$ are sufficiently large). Clearly, $\lambda_{a}$ and $\delta_{a}$ are functions of the period length-when one period is long, an incumbent is less likely to survive towards the end of a period, and an outsider is more likely to enter before the end of a period. Thus, potentially interesting dynamics driven by large values $\lambda_{a}$ and $\delta_{a}$ are essentially an artifact of the assumption that entry and exit occur only between periods. Given that entry and exit occur continuously in reality, this type of dynamics is not realistically relevant.

With $\gamma_{a} \geq 0$, the convergence to the steady state is monotone and stable when the same policy is chosen over a period of time - when the same policy $a$ is chosen forever, the long-run value of $L_{t}$ monotonically converges to $\bar{L}_{a}$.

\subsection{Equilibrium}

An important feature of the dynamic environment is that the status of each firm as a leader or a follower changes over time. Moreover, since we assume that transition events (conditional on regime) are independent across firms, the set of firms which share the common status at a given date will be different and each firm may have different preferences over policy regimes at a later period. Therefore, the interests of a set of firms are guaranteed to be aligned for one period only.

We assume that, at each period, firms with the same status (and hence identical interests over policy choices) form a coalition (special interest group) in order to advance their interests. We do not investigate the coalition formation process explicitly and we assume symmetric outcomes within a coalition. At the end of the period, the coalition is dissolved. Each coalition acts to maximize the present discounted value of the profit for its representative member.

We will concentrate on symmetric Markov equilibria. This means (i) we assume that strategies do not depend on the identity of the firms, but only on their status as a leader

or a follower; and (ii) strategies can be conditioned only on payoff-relevant variables, which in our case include only the mass of leaders in the economy, $L_{t}$, and the firm's own state of being a leader or a follower.

Let us call (consistently with the notation in the static model) the equilibrium present 
discounted value of the net payoff (profit minus the payment to the policymaker) for a firm of type $i(i=\ell, f) n_{i}(L)$. Similarly, we denote the equilibrium present discounted value of the gross payoff to firm of type $i(i=\ell, f)$ from the political action $a(a=e, b)$ as $v_{i}(a, L)$. For the leaders, it satisfies the following equation:

$$
v_{\ell}(a, L)=\left(1-\delta_{a}\right)\left(\pi_{\ell}+\beta n_{\ell}(\mathcal{L}(a, L))\right)+\delta_{a} \beta n_{f}(\mathcal{L}(a, L))
$$

and for the followers the following equation is satisfied:

$$
v_{f}(a, L)=\lambda_{a}\left(\pi_{f}+\beta n_{\ell}(\mathcal{L}(a, L))\right)+\left(1-\lambda_{a}\right) \beta n_{f}(\mathcal{L}(a, L)) .
$$

Then the total gross value of the coalition is simply $V_{\ell}(a, L)=L v_{\ell}(a, L)$ and $V_{f}(a, L)=$ $(1-L) v_{f}(a, L)$.

As in the static model, the coalition chooses the menu of the payment $R_{i}(a ; L)$ in order to maximize $V_{i}(a, L)-R_{i}(a, L)$. We assume that the coalition can commit to the payment menu only for the current period. ${ }^{7}$

The individual equilibrium net value $n_{i}(L)$ is then given by:

$$
n_{\ell}(L)=\frac{V_{\ell}\left(a^{*}(L), L\right)-R_{\ell}\left(a^{*}(L), L\right)}{L}
$$

and

$$
n_{f}(L)=\frac{V_{f}\left(a^{*}(L), L\right)-R_{f}\left(a^{*}(L), L\right)}{1-L},
$$

where $a^{*}(L)$ is the equilibrium policy action. Thus, if we define $r_{\ell}(a, L) \equiv R_{\ell}(a, L) / L$ and $r_{f}(a, L) \equiv R_{f}(a, L) /(1-L)$, the equilibrium payoffs satisfy the functional equations

$$
n_{\ell}(L)=\left(1-\delta_{a^{*}(L)}\right)\left(\pi_{\ell}+\beta n_{\ell}\left(\mathcal{L}\left(a^{*}(L), L\right)\right)\right)+\delta_{a^{*}(L)} \beta n_{f}\left(\mathcal{L}\left(a^{*}(L), L\right)\right)-r_{\ell}\left(a^{*}(L), L\right)
$$

and

$$
n_{f}(L)=\lambda_{a^{*}(L)}\left(\pi_{f}+\beta n_{\ell}\left(\mathcal{L}\left(a^{*}(L), L\right)\right)\right)+\left(1-\lambda_{a^{*}(L)}\right) \beta n_{f}\left(\mathcal{L}\left(a^{*}(L), L\right)\right)-r_{f}\left(a^{*}(L), L\right) .
$$

\footnotetext{
${ }^{7}$ Note that maximizing the total net value of the coalition is equivalent to maximizing the net value of a representative member of the coalition.
} 
The policymaker's present discounted value of the receipt of the payment is denoted by $G\left(L_{t}\right)$. The Bellman equation for the policymaker is

$$
G(L)=\max _{a}\left\{R_{\ell}(a ; L)+R_{f}(a ; L)+\beta G(\mathcal{L}(a, L))\right\}
$$

Below, we follow Bergemann and Välimäki (2003) in extending Bernheim and Whinston's (1986) equilibrium concepts to a dynamic situation. In particular, we consider the Markov Perfect Equilibrium as the baseline equilibrium concept and define the Truthful Markov Perfect Equilibrium as the refinement we utilize.

\subsubsection{Truthful Markov Perfect Equilibrium}

This section defines and describes the Truthful Markov Perfect Equilibrium in our setting. As we noted earlier, we focus on Markov strategies in the sense that the actions of firms and the policymaker depend only on the payoff-relevant state variables. Here, the payoffrelevant state for a firm is only $L$, aside from whether one is a leader or a follower. For the policymaker, the payoff-relevant state is only $L$. The definition of the truthful Markov strategy is similar to the static case in Definition 1.

Definition $3 R_{i}(a ; L)$ (for $i=\ell, f$ ) is said to be a truthful Markov strategy relative to $(\hat{a}, L)$ if, for $a=e, b$, either

$$
N_{i}(a ; L)=N_{i}(\hat{a} ; L)
$$

or

$$
N_{i}(a ; L)<N_{i}(\hat{a} ; L) \text { and } R_{i}(\hat{a} ; L)=0
$$

holds.

The Truthful Markov Perfect Equilibrium is also a natural extension of the Truthful Nash Equilibrium in Definition 2.

Definition 4 Let $\mathcal{R}(L) \equiv\left\{R_{i}(a ; L)\right\}_{i=\ell, f ; a=e, b} .(\mathcal{R}(L), \hat{a}(\mathcal{R}(L) ; L))$ is a Markov Perfect Equilibrium if 
(i) For all $L$ and $\mathcal{R}^{\prime}(L) \equiv\left\{R_{i}^{\prime}(a ; L)\right\}_{i=\ell, f ; a=e, b}, \hat{a}\left(\mathcal{R}^{\prime}(L) ; L\right)$ is a solution to

$$
\max _{a \in\{e, b\}} R_{\ell}^{\prime}(a ; L)+R_{f}^{\prime}(a ; L)+\beta G(\mathcal{L}(a, L)),
$$

and

(ii) for each $i$ and all $L$, there is no other $R_{i}^{\prime}(a ; L)$ such that

$$
V_{i}(\tilde{a} ; L)-R_{i}^{\prime}(\tilde{a} ; L)>V_{i}(a ; L)-R_{i}(a ; L),
$$

where $\tilde{a}=\hat{a}(\tilde{\mathcal{R}}(L) ; L)), \tilde{\mathcal{R}}(L) \equiv\left\{R_{i}^{\prime}(a ; L), R_{-i}(a ; L)\right\}_{a=e, b}$, and $\left.a=\hat{a}(\mathcal{R}(L) ; L)\right)$.

(iii) $V_{i}(a, L)$ and $G(L)$ satisfy the Bellman equations above for $\mathcal{R}(L)$.

It is called a Truthful Markov Perfect Equilibrium if it is a Markov Perfect Equilibrium and each coalition plays a truthful Markov strategy relative to $(\hat{a}(\mathcal{R}(L) ; L), L)$.

We define

$$
a^{*}(L) \equiv \hat{a}(\mathcal{R}(L) ; L)
$$

where $\mathcal{R}(L)$ is the equilibrium reward (payment) function as above, and call $a^{*}(L)$ as the equilibrium political outcome when the state is $L$.

Proposition 2 below describes a Truthful Markov Perfect Equilibrium (if it exists - we will discuss the issue of existence in Section 3.2.2) in our economy. It can easily be checked with the definition, and thus the proof is omitted. There are two cases, called the "Regular Case" and the "Irregular Case." The Regular Case corresponds to the one analogous to the static model - the leader coalition prefers "blocking" and the follower coalition prefers "free entry." In the Irregular Case, the dynamic forces (through future values) overturn one (or both) coalition's policy preferences. The leader coalition might like the free entry policy (Irregular Case I), the follower coalition might like the entry blocking policy (Irregular Case II), or both (Irregular Case III).$^{8}$

\section{Proposition 2 The following is a Truthful Markov Perfect Equilibrium:}

\footnotetext{
${ }^{8}$ In the numerical examples computed in Section 3.4, the equilibrium turns out to be the "Regular Case" for all $L$.
} 
Regular Case: $V_{\ell}(b ; L) \geq V_{\ell}(e ; L)$ and $V_{f}(e ; L) \geq V_{f}(b ; L)$

- If $V_{\ell}(b ; L)+V_{f}(b ; L)+\beta G\left(L_{b}^{\prime}\right) \geq V_{\ell}(e ; L)+V_{f}(e ; L)+\beta G\left(L_{e}^{\prime}\right), a^{*}(L)=b$ is selected.

- Case I: If $V_{f}(e ; L)-V_{f}(b ; L)+\beta\left[G\left(L_{e}^{\prime}\right)-G\left(L_{b}^{\prime}\right)\right] \geq 0$,

$$
\begin{gathered}
\left(R_{\ell}(e ; L), R_{\ell}(b ; L)\right)=\left(0, V_{f}(e ; L)-V_{f}(b ; L)+\beta\left[G\left(L_{e}^{\prime}\right)-G\left(L_{b}^{\prime}\right)\right]\right) \\
\left(R_{f}(e ; L), R_{f}(b ; L)\right)=\left(V_{f}(e ; L)-V_{f}(b ; L), 0\right) .
\end{gathered}
$$

- Case II: Otherwise (note that $G\left(L_{b}^{\prime}\right)>G\left(L_{e}^{\prime}\right)$ in this case since $V_{f}(e ; L)>$ $\left.V_{f}(b ; L)\right)$,

$$
\begin{gathered}
\left(R_{\ell}(e ; L), R_{\ell}(b ; L)\right)=(0,0) \\
\left(R_{f}(e ; L), R_{f}(b ; L)\right)=\left(V_{f}(e ; L)-V_{f}(b ; L), 0\right) .
\end{gathered}
$$

- Otherwise, $a^{*}(L)=e$ is selected.

- Case I: If $V_{\ell}(b ; L)-V_{\ell}(e ; L)+\beta\left[G\left(L_{b}^{\prime}\right)-G\left(L_{e}^{\prime}\right)\right] \geq 0$,

$$
\begin{gathered}
\left(R_{\ell}(e ; L), R_{\ell}(b ; L)\right)=\left(0, V_{\ell}(b ; L)-V_{\ell}(e ; L)\right) \\
\left(R_{f}(e ; L), R_{f}(b ; L)\right)=\left(V_{\ell}(b ; L)-V_{\ell}(e ; L)+\beta\left[G\left(L_{b}^{\prime}\right)-G\left(L_{e}^{\prime}\right)\right], 0\right) .
\end{gathered}
$$

- Case II: Otherwise (note that $G\left(L_{e}^{\prime}\right)>G\left(L_{b}^{\prime}\right)$ in this case since $V_{\ell}(b ; L)>$ $\left.V_{\ell}(e ; L)\right)$.

$$
\begin{gathered}
\left(R_{\ell}(e ; L), R_{\ell}(b ; L)\right)=\left(0, V_{\ell}(b ; L)-V_{\ell}(e ; L)\right) \\
\left(R_{f}(e ; L), R_{f}(b ; L)\right)=(0,0) .
\end{gathered}
$$

Irregular Case I: $V_{\ell}(b ; L)<V_{\ell}(e ; L)$ and $V_{f}(e ; L) \geq V_{f}(b ; L)$

- If $V_{\ell}(b ; L)+V_{f}(b ; L)+\beta G\left(L_{b}^{\prime}\right) \geq V_{\ell}(e ; L)+V_{f}(e ; L)+\beta G\left(L_{e}^{\prime}\right), a^{*}(L)=b$ is selected.

$$
\begin{gathered}
\left(R_{\ell}(e ; L), R_{\ell}(b ; L)\right)=\left(V_{\ell}(e ; L)-V_{\ell}(b ; L), 0\right), \\
\left(R_{f}(e ; L), R_{f}(b ; L)\right)=\left(V_{f}(e ; L)-V_{f}(b ; L), 0\right) .
\end{gathered}
$$


- Otherwise, $a^{*}(L)=e$ is selected.

$$
\begin{aligned}
& \left(R_{\ell}(e ; L), R_{\ell}(b ; L)\right)=\left(R_{1}, 0\right), \\
& \left(R_{f}(e ; L), R_{f}(b ; L)\right)=\left(R_{2}, 0\right),
\end{aligned}
$$

where $V_{\ell}(e ; L)-V_{\ell}(b ; L) \geq R_{1} \geq 0, V_{f}(e ; L)-V_{f}(b ; L) \geq R_{2} \geq 0$, and $R_{1}+R_{2}=$ $\beta\left[G\left(L_{b}^{\prime}\right)-G\left(L_{e}^{\prime}\right)\right]$.

Irregular Case II: $V_{\ell}(b ; L) \geq V_{\ell}(e ; L)$ and $V_{f}(e ; L)<V_{f}(b ; L)$

- If $V_{\ell}(b ; L)+V_{f}(b ; L)+\beta G\left(L_{b}^{\prime}\right) \geq V_{\ell}(e ; L)+V_{f}(e ; L)+\beta G\left(L_{e}^{\prime}\right), a^{*}(L)=b$ is selected.

$$
\begin{aligned}
& \left(R_{\ell}(e ; L), R_{\ell}(b ; L)\right)=\left(0, R_{1}\right), \\
& \left(R_{f}(e ; L), R_{f}(b ; L)\right)=\left(0, R_{2}\right),
\end{aligned}
$$

where $V_{\ell}(b ; L)-V_{\ell}(e ; L) \geq R_{1} \geq 0, V_{f}(b ; L)-V_{f}(e ; L) \geq R_{2} \geq 0$, and $R_{1}+R_{2}=$ $\beta\left[G\left(L_{e}^{\prime}\right)-G\left(L_{b}^{\prime}\right)\right]$

- Otherwise, $a^{*}(L)=e$ is selected.

$$
\begin{aligned}
\left(R_{\ell}(e ; L), R_{\ell}(b ; L)\right) & =\left(0, V_{\ell}(b ; L)-V_{\ell}(e ; L)\right) \\
\left(R_{f}(e ; L), R_{f}(b ; L)\right) & =\left(0, V_{f}(b ; L)-V_{f}(e ; L)\right) .
\end{aligned}
$$

Irregular Case III: $V_{\ell}(b ; L)<V_{\ell}(e ; L)$ and $V_{f}(e ; L)<V_{f}(b ; L)$

- If $V_{\ell}(b ; L)+V_{f}(b ; L)+\beta G\left(L_{b}^{\prime}\right) \geq V_{\ell}(e ; L)+V_{f}(e ; L)+\beta G\left(L_{e}^{\prime}\right), a^{*}(L)=b$ is selected.

- Case I: If $V_{\ell}(e ; L)-V_{\ell}(b ; L)+\beta\left[G\left(L_{e}^{\prime}\right)-G\left(L_{b}^{\prime}\right)\right] \geq 0$,

$$
\begin{gathered}
\left(R_{\ell}(e ; L), R_{\ell}(b ; L)\right)=\left(V_{\ell}(e ; L)-V_{\ell}(b ; L), 0\right), \\
\left(R_{f}(e ; L), R_{f}(b ; L)\right)=\left(0, V_{\ell}(e ; L)-V_{\ell}(b ; L)+\beta\left[G\left(L_{e}^{\prime}\right)-G\left(L_{b}^{\prime}\right)\right]\right) .
\end{gathered}
$$


- Case II: Otherwise (note that $G\left(L_{b}^{\prime}\right)>G\left(L_{e}^{\prime}\right)$ in this case since $V_{\ell}(e ; L)>$ $\left.V_{\ell}(b ; L)\right)$,

$$
\begin{gathered}
\left(R_{\ell}(e ; L), R_{\ell}(b ; L)\right)=\left(0, V_{\ell}(e ; L)-V_{\ell}(b ; L)\right) \\
\left(R_{f}(e ; L), R_{f}(b ; L)\right)=(0,0) .
\end{gathered}
$$

- Otherwise, $a^{*}(L)=e$ is selected.

- Case I: If $V_{f}(b ; L)-V_{f}(e ; L)+\beta\left[G\left(L_{b}^{\prime}\right)-G\left(L_{e}^{\prime}\right)\right] \geq 0$,

$$
\begin{gathered}
\left(R_{\ell}(e ; L), R_{\ell}(b ; L)\right)=\left(V_{f}(b ; L)-V_{f}(e ; L)+\beta\left[G\left(L_{b}^{\prime}\right)-G\left(L_{e}^{\prime}\right)\right], 0\right), \\
\left(R_{f}(e ; L), R_{f}(b ; L)\right)=\left(0, V_{f}(b ; L)-V_{f}(e ; L)\right) .
\end{gathered}
$$

- Case II: Otherwise (note that $G\left(L_{e}^{\prime}\right)>G\left(L_{b}^{\prime}\right)$ in this case since $V_{f}(b ; L)>$ $\left.V_{f}(e ; L)\right)$.

$$
\begin{gathered}
\left(R_{\ell}(e ; L), R_{\ell}(b ; L)\right)=(0,0), \\
\left(R_{f}(e ; L), R_{f}(b ; L)\right)=\left(0, V_{f}(b ; L)-V_{f}(e ; L)\right) .
\end{gathered}
$$

Next, we show the existence of the equilibrium before characterizing the equilibrium. Unfortunately, since contraction arguments cannot be used, the normal techniques to prove existence of equilibrium are unavailable when the state variable $L$ is continuous. However, the continuous state variable has a benefit of allowing us simple and tractable characterizations of the equilibrium policy action $a^{*}(L)$ as we will see below. In the following section we introduce a discrete version of the economy, in which we prove (following Bergemann and Välimäki (2003)) that an equilibrium exists. Later on (in Section 3.3.3), we will show that the equilibrium policy action in the discrete-state economy converges to the one in the continuousstate economy as the discrete states become "finer" and approach a continuum.

\subsubsection{Existence of discrete-state equilibrium}

Below we will prove the existence of the equilibrium by modifying the proof by Bergemann and Välimäki (2003). There are two differences between our model and the model considered by Bergemann and Välimäki (2003): 
(i) Bergemann and Välimäki (2003) consider a model with finite states, while in our model one of the state variables $(L)$ is continuous.

(ii) In Bergemann and Välimäki (2003), the identity of each principal (the coalitions in our model) is unchanged, while in our model it changes over time. (A firm can be in the leader coalition today and in the follower coalition tomorrow.)

As we note above, the difference (i) forces us first to start from considering a model with discrete state variables that approximates our original model. In this section, we establish the existence of the equilibrium in this discrete-state economy. In order to prove it, we still need to modify the proof of Bergemann and Välimäki (2003) because of the above difference (ii).

Consider a discrete-state version of our economy as in the following. There are $m$ firms in the economy. We normalize the population and assume that each firm has a mass $1 / m$, so that the total mass is one. ${ }^{9}$ Denote the mass of leaders when there are $i$ leaders in the economy as $L^{i} \equiv i / m$. At the beginning of each period, the status of every firm as a leader or a follower is publicly known; therefore the state of the economy is an $m$-dimensional vector of firm states $\omega$. We denote the space of the states as $\Omega$. Clearly the cardinality of $\Omega$ is $2^{m}$. As before, the probability that a leader in the current period will remain a leader is given by $1-\delta_{a}$, where $a$ is the policy regime; similarly the probability that a follower will switch to a leader is $\lambda_{a}$. We assume that the transitions are independent across firms.

If we condition on the number of leaders in the current period and on the policy regime, the number of leaders in the following period is a sum of a discrete and finite number of independent random variables with nonzero variance. Thus (unlike the continuum case) the number of leaders in the next period is stochastic. It is a sum of two random variables (the number of leaders that remained leaders and the number of followers that switched) which follow binomial distributions.

\footnotetext{
${ }^{9}$ Later on, we take a limit of $m \rightarrow \infty$. The equilibrium strategies and payoffs clearly depend on $m$. To simplify the notation, here we suppress this dependence.
} 
In order to construct the transition probabilities for the aggregate state, first consider a subgroup of firms (that can include both leaders and followers) and let the number of firms in the subgroup be $k$. Define $Q_{i, j}^{k}(a)$ to be the probability that this subgroup, which has $i$ leaders in the current period, will have $j$ leaders in the following period when the current period policy is $a$ :

$Q_{i, j}^{k}(a)= \begin{cases}\sum_{\substack{h=\max \{j-(k-i), 0\} \\ 0}}^{\min \{i, j\}}\left(\begin{array}{l}i \\ h\end{array}\right)\left(1-\delta_{a}\right)^{h} \delta_{a}^{i-h}\left(\begin{array}{l}k-i \\ j-h\end{array}\right) \lambda_{a}^{j-h}\left(1-\lambda_{a}\right)^{k-i-(j-h)} & \text { if } 0 \leq j \leq k, \\ \text { otherwise. }\end{cases}$

The first line of (13) represents the transition probability for a case that is possible to happen (there cannot be more than $k$ leaders in this subgroup at any period). Each term in the first line consists of two parts. The first part is the probability that $h$ firms among the current $i$ leaders remain leaders next period. The second part is the probability that $j-h$ firms among the current $k-j$ followers become leaders next period. The possible values for $h$ run from 0 to $j$. If $j$ is larger than the current number of followers (that is, $j \geq k-i$ ), some of the leaders must remain leaders to reach $j$ in total, and thus $h \geq j-(k-i)$. Also note that $h$ cannot exceed $i$, and if $j \geq i$ some of the followers must become leaders. Therefore, the possible values for $h$ range from $\max \{j-(k-i), 0\}$ to $\min \{i, j\}$. Let $P_{i, j}(a)$ be the conditional probability that there are $j$ leaders in the entire economy in period $t+1$ (that is, $L_{t+1}=L^{j}$ ) when there are $i$ leaders at time $t$ (that is, $L_{t}=L^{i}$ ) and the time- $t$ policy is a. Then, $P_{i, j}(a)$ is given by $P_{i, j}(a)=Q_{i, j}^{m}(a)$.

We assume that, at the beginning of the period, all leaders form a "leader coalition" and all followers form a "follower coalition" in order to pursue their respective collective interests. We do not investigate the process of coalition formation and multilateral bargaining inside the coalition, and will only look at symmetric outcomes. Firms can commit to the collective action only for one period and condition their strategies only on payoff-relevant variables (Markovian assumption). Since all firms have the same transition probabilities and are treated symmetrically, the coalition maximizes the expected discounted payoff of the representative firm in each coalition. The symmetry assumptions imply that the only 
payoff-relevant aggregate variable for a firm is the number of leaders $i$ or, equivalently, the mass of leaders $L^{i}(=i / m)$. As in our baseline model, let $n_{s}\left(L^{i}\right)$ be the expected payoff of firm type $s(s=\ell, f)$ if there is $L^{i}$ mass of leaders; let $v_{s}\left(a, L^{i}\right)$ be the corresponding gross (before the payment to the policymaker) payoff.

Then $v_{s}\left(a, L^{i}\right)$ can be expressed as

$$
v_{\ell}\left(a, L^{i}\right)=\left(1-\delta_{a}\right) \pi_{\ell}+\beta\left(1-\delta_{a}\right) \sum_{j=1}^{m} Q_{i-1, j-1}^{m-1}(a) n_{\ell}\left(L^{j}\right)+\beta \delta_{a} \sum_{j=0}^{m-1} Q_{i-1, j}^{m-1}(a) n_{f}\left(L^{j}\right)
$$

for a leader and

$$
v_{f}\left(a, L^{i}\right)=\lambda_{a} \pi_{f}+\beta \lambda_{a} \sum_{j=1}^{m} Q_{i, j-1}^{m-1}(a) n_{\ell}\left(L^{j}\right)+\beta\left(1-\lambda_{a}\right) \sum_{j=0}^{m-1} Q_{i, j}^{m-1}(a) n_{f}\left(L^{j}\right)
$$

for a follower. Equations (14) and (15) are the discrete versions of (7) and (8). The values for the entire coalitions are $V_{f}\left(a, L^{i}\right)=v_{f}\left(a, L^{i}\right)\left(1-L^{i}\right)$ and $V_{\ell}\left(a, L^{i}\right)=v_{\ell}\left(a, L^{i}\right) L^{i}$, similarly to the baseline (continuous-state) model. Denoting the payment to the policymaker from coalition $s(s=\ell, f)$ as $R_{s}\left(a, L^{i}\right)$ and the individual payments as $r_{\ell}\left(a, L^{i}\right) \equiv R_{\ell}\left(a, L^{i}\right) / L^{i}$ and $r_{f}\left(a, L^{i}\right) \equiv R_{f}\left(a, L^{i}\right) /\left(1-L^{i}\right.$ ) (with value zero when the corresponding population is zero), $n_{s}\left(L^{i}\right)$ can be defined in the same manner as (9) and (10). Then the Truthful Markov Perfect Equilibrium in the discrete economy is defined in essentially the same manner as in Definition 3. Proposition 3 below shows that the Truthful Markov Perfect Equilibrium exists. Moreover, the political outcome $a^{m *}\left(L^{i}\right)$ is equivalent to the solution that maximizes the discounted present value (denoted $S^{m}\left(L^{i}\right)$ in Proposition 3 below) of total profit (surplus) in each period $L^{i}\left(1-\delta_{a}\right) \pi_{\ell}+\left(1-L^{i}\right) \lambda_{a} \pi_{f}$. In the next section, we show that this equivalence holds even when $L$ is continuous, and use this fact extensively in characterizing the Truthful Markov Perfect Equilibrium in Section 3.3.2 and Section 3.4.

Proposition 3 A Truthful Markov Perfect Equilibrium exists. Moreover, the equilibrium action profile $a^{m *}\left(L^{i}\right)$ is the maximizer in the following Bellman equation:

$$
S^{m}\left(L^{i}\right)=\max _{a \in\{b, e\}}\left\{L^{i}\left(1-\delta_{a}\right) \pi_{\ell}+\left(1-L^{i}\right) \lambda_{a} \pi_{f}+\beta \sum_{j=0}^{m} P_{i, j}(a) S^{m}\left(L^{j}\right)\right\} .
$$


Proof. See Appendix B.

In Section 3.3.3, we will formally show that the political outcome from this discrete economy converges to the one with the continuous state variable $L$ as $m \rightarrow \infty$. In the next section, we turn back to the model with the continuous state variable and characterize the equilibrium outcome.

\subsection{Characterizing the political outcome}

In this section, we characterize a general property of the equilibrium policies $a^{*}(L)$. We show that, similarly to the static model, $a^{*}(L)$ exhibits a threshold property: "free entry" is chosen when $L$ is small and "blocking" is chosen when $L$ is larger than a certain threshold.

\subsubsection{Equivalence to surplus maximizing}

As in Proposition 3, it turns out that the political outcome $a^{*}(L)$ is equivalent to the solution that maximizes the discounted present value of total profit (surplus) $L\left(1-\delta_{a}\right) \pi_{\ell}+(1-L) \lambda_{a} \pi_{f}$. This can be shown directly using the equilibrium outcome in Proposition 2.

Proposition 4 The equilibrium policy choice $a^{*}(L)$ is the policy function for the following dynamic programming problem:

$$
S(L)=\max _{a \in\{b, e\}}\left\{\left(1-\delta_{a}\right) L \pi_{\ell}+\lambda_{a}(1-L) \pi_{f}+\beta S(\mathcal{L}(a, L))\right\} .
$$

Proof. See Appendix B.

We will use this property extensively below when we characterize the properties of the equilibrium. Here we emphasize that the fact that the policy maximizes the present-value of total surplus does not mean that it is "optimal." In particular, consumers do not participate in the political process and the welfare for the consumers is not considered when calculating the "total surplus." In our model, the consumers are not explicitly considered since we focus on the positive analysis of equilibrium policymaking. However, as we discussed in the introduction, it is likely that the consumer would benefit from the entry of new firms ("creative 
destruction"). Thus, if entry blocking is chosen as the equilibrium policy, it is likely that this policy choice is very costly when we consider the economy-wide welfare including the consumers.

The following proposition establishes some general properties of the total surplus function $S(L) . S(L)$ satisfies the Bellman equation (17), which implies that $S$ can be analyzed with standard recursive methods. Let the mapping in functional space defined by the righthand side of (17) be denoted by $T$. Since the per-period surplus falls in the compact set $\left[0, \max \left\{\pi_{\ell}, \pi_{f}\right\}\right], T$ is a contraction. Hence equation (17) has a unique solution.

Proposition 5 The operator $T$ is a contraction. It has a unique continuous and convex fixed point. The unique fixed point $S$ is differentiable everywhere, except on a set of points that is at most countable. Moreover,

$$
S^{\prime}(L) \in\left[\min _{a \in\{\ell, f\}} \frac{\pi_{\ell}-\delta_{a} \pi_{\ell}-\lambda_{a} \pi_{f}}{1-\beta \gamma_{a}}, \max _{a \in\{\ell, f\}} \frac{\pi_{\ell}-\delta_{a} \pi_{\ell}-\lambda_{a} \pi_{f}}{1-\beta \gamma_{a}}\right]
$$

for all $L$ where the derivative is defined.

Proof. See Appendix B.

\subsubsection{Results: Threshold rule}

In this section, we show that the equilibrium outcome for $a^{*}(L)$ is a threshold rule, similar to the static case. It requires some extra preparations in order to show the result formally.

First, let us introduce some notation. Let $p_{a} \equiv \pi_{\ell}-\delta_{a} \pi_{\ell}-\lambda_{a} \pi_{f}$ and $q_{a} \equiv \lambda_{a} \pi_{f}$. Let $S_{a}(L)$ be the discounted sum of surplus if the economy starts at $L$ and the policy $a$ is followed forever. It is easy to see that

$$
S_{a}(L)=\frac{p_{a} \bar{L}_{a}+q_{a}}{1-\beta}+\frac{p_{a}\left(L-\bar{L}_{a}\right)}{1-\beta \gamma_{a}}
$$

and

$$
S_{a}(L)=p_{a} L+q_{a}+\beta S_{a}(\mathcal{L}(a, L))
$$

hold. 
Let $S_{a, a^{\prime}}(L)$ denote the discounted sum of surplus if the economy starts at $L$, chooses $a$ for the first period and $a^{\prime}$ thereafter. Then

$$
S_{a, a^{\prime}}(L)=p_{a} L+q_{a}+\beta S_{a^{\prime}}(\mathcal{L}(a, L))
$$

holds. The following proposition characterizes the threshold rules.

Proposition 6 The optimal policy is determined as follows:

1. If $p_{e} /\left(1-\beta \gamma_{e}\right) \geq p_{b} /\left(1-\beta \gamma_{b}\right)$, then the optimal choice correspondence satisfies $e \in a^{*}(L)$ for all $L$.

2. If $p_{e} /\left(1-\beta \gamma_{e}\right)<p_{b} /\left(1-\beta \gamma_{b}\right)$ and $\bar{L}_{e} \leq \bar{L}_{b}$, then there exists some $L^{*}$ such that $e \in a^{*}(L)$ for all $L<L^{*}, b \in a^{*}(L)$ for all $L>L^{*}$, and $\{e, b\}=a^{*}\left(L^{*}\right)$.

(a) If $S_{b}\left(\bar{L}_{e}\right) \geq S_{e, b}\left(\bar{L}_{e}\right)$, either $L^{*}<0$ or $L^{*}$ solves the equation $S_{b}\left(L^{*}\right)=S_{e, b}\left(L^{*}\right)$ and $L^{*} \leq \bar{L}_{e}$.

(b) If $S_{e}\left(\bar{L}_{b}\right) \geq S_{b, e}\left(\bar{L}_{b}\right)$, either $L^{*}>1$ or $L^{*}$ solves the equation $S_{e}\left(L^{*}\right)=S_{b, e}\left(L^{*}\right)$ and $L^{*} \geq \bar{L}_{b}$.

(c) In all other cases, $L^{*}$ solves the equation $S_{b}\left(L^{*}\right)=S_{e}\left(L^{*}\right)$ and $L^{*} \in\left(\bar{L}_{e}, \bar{L}_{b}\right)$.

3. If $p_{e} /\left(1-\beta \gamma_{e}\right)<p_{b} /\left(1-\beta \gamma_{b}\right), \bar{L}_{b}<\bar{L}_{e}$, and

(a) $S_{b}\left(\bar{L}_{b}\right) \geq S_{e, b}\left(\bar{L}_{b}\right)$, then $e \in a^{*}(L)$ for all $L<L^{*}, b \in a^{*}(L)$ for all $L>L^{*}$ and $\{e, b\}=a^{*}\left(L^{*}\right)$, where either $L^{*}<0$ or $L^{*}$ solves the equation $S_{b}\left(L^{*}\right)=S_{e, b}\left(L^{*}\right)$ and $L^{*} \leq \bar{L}_{b}$.

(b) $S_{e}\left(\bar{L}_{e}\right) \geq S_{b, e}\left(\bar{L}_{e}\right)$, then $e \in a^{*}(L)$ for all $L<L^{*}, b \in a^{*}(L)$ for all $L>L^{*}$ and $\{e, b\}=a^{*}\left(L^{*}\right)$ where either $L^{*}>1$ or $L^{*}$ solves the equation $S_{e}\left(L^{*}\right)=S_{b, e}\left(L^{*}\right)$ and $L^{*} \geq \bar{L}_{e}$.

(c) If neither of the conditions in (a) or (b) are satisfied and $1-\beta \gamma_{e}-\beta \gamma_{b}>0$, then there exists some $L^{*} \in\left(\bar{L}_{b}, \bar{L}_{e}\right)$ such that $e \in a^{*}(L)$ for all $L<L^{*}, b \in a^{*}(L)$ for all $L>L^{*}$ and $\{e, b\}=a^{*}\left(L^{*}\right)$. 


\section{Proof. See Appendix B.}

In all above cases, the political decision follows a threshold rule: $a^{*}(L)=e$ when $L$ is small and $a^{*}(L)=b$ when $L$ is large. (Case 1 can be considered a "corner solution" for the threshold.)

In the third statement, there are some cases (when both $S_{b}\left(\bar{L}_{b}\right) \geq S_{e, b}\left(\bar{L}_{b}\right)$ and $S_{e}\left(\bar{L}_{e}\right) \geq$ $S_{b, e}\left(\bar{L}_{e}\right)$ do not hold) in which we have not been able to prove the threshold property in general. As we will see later, this corresponds to the situation where $L$ exhibits cyclical dynamics. For this case, we have solved the model numerically and experimented with many parameter values, including the ones outside the parametric restriction. In all our experiments, $a^{*}(L)$ turned out to possess a threshold property as in all the other situations.

\subsubsection{Convergence from discrete-state model to continuous-state model}

In the previous section, we characterized the political equilibrium in the model with continuous state variable $L$. As we have just seen, the continuous state case has an advantage that it is relatively easy to characterize the equilibrium outcomes, and therefore in most of this paper we focus on the model with the continuous state. Due to technical difficulties, however, we were not able to directly show existence of equilibrium when the state variable is continuous. In Section 3.2.2 we developed a version of the model with a discrete number of firms, and showed that a Markov Perfect Equilibrium in Truthful Strategies exists in that environment. Thus it is useful to establish that as the discrete grids of $L$ become "finer," the equilibrium outcome of the discrete-state model converges to the continuous-state model. This allows us to view the continuous-state model as approximating a discrete-state model with many possible values of $L$, in which the existence of an equilibrium is guaranteed.

Below, we show the convergence in terms of total surplus and equilibrium policy functions. Proposition 7 below establishes the convergence of the total surplus function.

Proposition 7 Let $S^{m}\left(L^{i}\right)$ and $S(L)$ be expected discounted total surplus functions for the discrete-state model and continuous-state model. $S^{m}\left(L^{i}\right)$ solves (16) and $S(L)$ solves (17). 
Then

$$
\lim _{m \rightarrow \infty} \max _{L^{i} \in\{0,1 / m, \ldots, 1\}}\left|S\left(L^{i}\right)-S^{m}\left(L^{i}\right)\right|=0
$$

holds. Here, $S\left(L^{i}\right)$ means the value of the $S(L)$ function evaluated at $L=L^{i}$.

Proof. See Appendix B.

Next we show that when Proposition 6 can be applied and thus the policy outcome has the threshold property, the policy outcome for the discrete-state economy $a^{m *}\left(L^{i}\right)$ converges to the policy outcome for the continuous-state model $a^{*}(L)$.

The intuition for the proof is that the loss from "taking the wrong action" increases as $L$ moves farther from $L^{*}$ (which is established in the proof of Proposition 6). With this fact and the fact that $S^{m}\left(L^{i}\right)$ converges to $S(L)$, we are able to show that the policy in the continuous-state model approximates the policy in the discrete-state model when the number of states is sufficiently large.

Proposition 8 Suppose that the (continuous-state) economy's environment is such that Proposition 6 can be applied. For the case of statement 1 in Proposition 6, there exists an $m^{* * *}$ such that if $m \geq m^{* * *}, a^{m *}\left(L^{i}\right)=e$ holds for all $L^{i} \in\{0,1 / m, \ldots, 1\}$. For the cases of statements 2 and 3 , let $L^{*}$ be the threshold. Then there exist bounds $\mathbf{L}_{l}^{m} \in[0,1]$ and $\mathbf{L}_{u}^{m} \in[0,1]$ such that $\mathbf{L}_{l}^{m}<L^{*}<\mathbf{L}_{u}^{m}$, where $a^{m *}\left(L_{i}\right)=e$ if $L_{i} \leq L_{l}^{m}$ and $a^{m *}\left(L^{i}\right)=b$ if $L_{i} \geq L_{u}^{m}$ for $L^{i} \in\{0,1 / m, \ldots, 1\}$. Moreover, $\lim _{m \rightarrow \infty} \mathbf{L}_{l}^{m}=\lim _{m \rightarrow \infty} \mathbf{L}_{u}^{m}=L^{*}$.

Proof. See Appendix B.

\subsection{Dynamics of the economy and the policy in some special cases}

In this section, we examine the dynamics of the economy in special cases and obtain sharper characterizations. In particular, we highlight some situations where the dynamics of the model economy provides insights on real-world phenomena. The dynamic paths of $L_{t}$ depend largely on the relative values of $L^{*}, \bar{L}_{e}$, and $\bar{L}_{b}$, and we divide the cases by the values of these 
variables. Note that $\bar{L}_{a}(a=e, b)$ is a function of $\lambda_{a}$ and $\delta_{a}$, while $L^{*}$ depends on all parameters of the model.

The dynamics of the model are determined by the relative position of the two steady states $\bar{L}_{e}, \bar{L}_{b}$ and the cutoff value $L^{*}$. The possibilities (discussed in detail below) are summarized by Figure 1. The reader may want to refer to the Figure as we go along with the discussion below.

\subsubsection{Political convergence and "British disease"}

When the threshold $L^{*}$ lies on the same side of $\bar{L}_{e}$ and $\bar{L}_{b}$ (that is, $L^{*} \leq \min \left\{\bar{L}_{e}, \bar{L}_{b}\right\}$ or $L^{*} \geq \max \left\{\bar{L}_{e}, \bar{L}_{b}\right\}, L$ converges to either $\bar{L}_{e}$ or $\bar{L}_{b}$ in the long run. This corresponds to 2(a)(b) and 3(a)(b) of Proposition 6 and the first four panels of Figure 1. We call this case

"political convergence," since the initial value of $L$ does not matter in the long run, and all economies with different starting values of $L$ (under the same fundamental parameter values) converge to the unique policy choice. Below we highlight two special cases.

\subsubsection{Political convergence I: Common steady state}

One special case in which political convergence always occurs is when $\bar{L}_{e}=\bar{L}_{b}$. Clearly, in this case $L^{*}$ is on the same side of $\bar{L}_{e}$ and $\bar{L}_{b}$. Call the common steady-state $\bar{L}$. Since $\bar{L}_{a}=\lambda_{a} /\left(\lambda_{a}+\delta_{a}\right), \bar{L}_{e}=\bar{L}_{b}$ if and only if $\lambda_{e} / \delta_{e}=\lambda_{b} / \delta_{b}$.

It is worthwhile to emphasize the comparison with the static outcome. Earlier we pointed out that both the intensive margin and extensive margin matter in the static model (see (5)). The difference in payoffs (3) and (4) provided the strengths of desire for the preferred strategy by the leader coalition and the follower coalition. Here, in the long run, the extensive margin is endogenous. The differences in the payoffs in the steady state are (from (7) and (8))

$$
V_{\ell}(b, \bar{L})-V_{\ell}(e, \bar{L})=\bar{L}\left(\delta_{e}-\delta_{b}\right)\left[\pi_{\ell}+\beta\left(n_{\ell}(\bar{L})-n_{f}(\bar{L})\right)\right]
$$

for the leader coalition and

$$
V_{f}(e, \bar{L})-V_{f}(e, \bar{L})=(1-\bar{L})\left(\lambda_{e}-\lambda_{b}\right)\left[\pi_{f}+\beta\left(n_{\ell}(\bar{L})-n_{f}(\bar{L})\right)\right]
$$


Convergence to $\bar{L}_{b}$ and $b$ regime

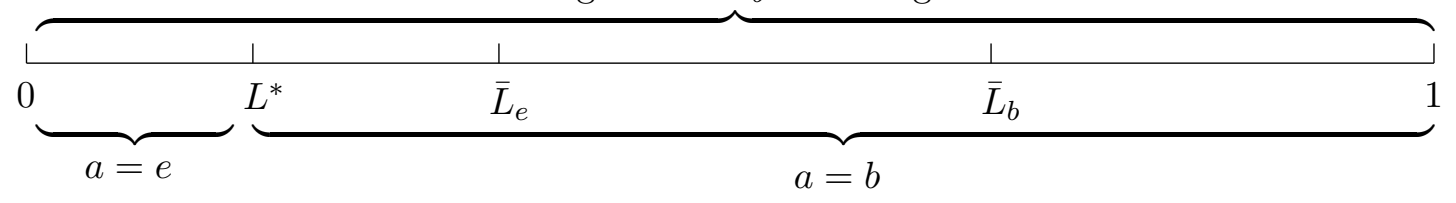

Convergence to $\bar{L}_{b}$ and $b$ regime

\begin{tabular}{|c|c|c|c|c|}
\hline L & 1 & 1 & 1 & 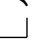 \\
\hline 0 & $L^{*}$ & $\bar{L}_{b}$ & $\bar{L}_{e}$ & 1 \\
\hline
\end{tabular}

Convergence to $\bar{L}_{e}$ and $e$ regime

\begin{tabular}{|c|c|c|c|c|}
\hline t & 1 & 1 & $\perp$ & 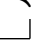 \\
\hline 0 & $\bar{L}_{e}$ & $\bar{L}_{b}$ & $L^{*}$ & 1 \\
\hline
\end{tabular}
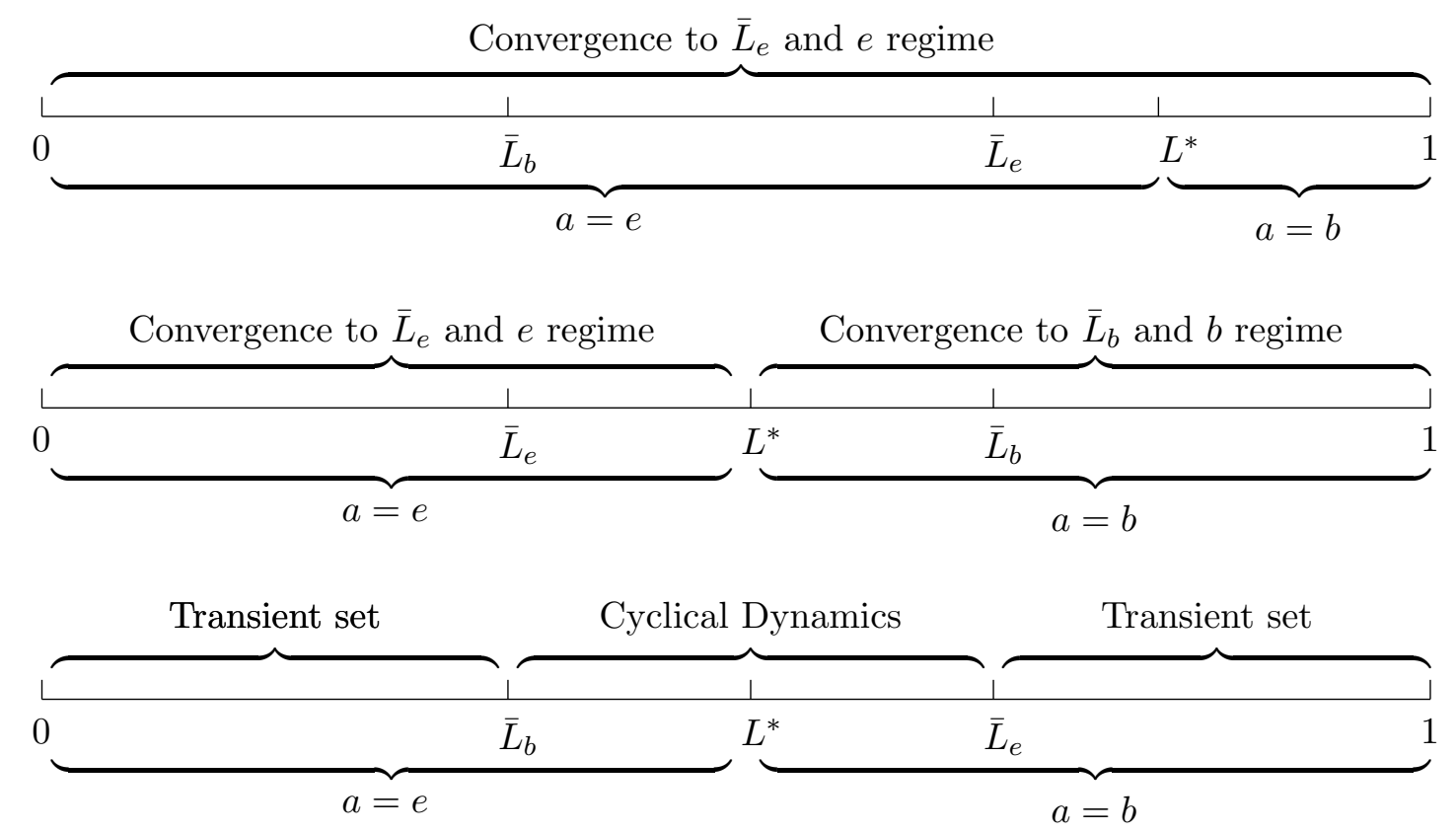

Figure 1: Possible dynamics. The mass of leaders $L$ is on the horizontal axis. The first four panels exhibit convergence in the long run. The case of multiple steady states is in the fifth panel and the case with cyclical dynamics is in the last panel. 
for the follower coalition. Since $G(L)$ is constant at $G(\bar{L})$ in the long run (and thus it does not depend on the policy choice), the policy determination is given by the comparison of these two differences, as in the static case. The changes from the static equations (3) and (4) are that here (i) there is the future payoff term $\beta\left(n_{\ell}(\bar{L})-n_{f}(\bar{L})\right)$ and (ii) $\bar{L}$ is determined by parameters $\lambda_{a}$ and $\delta_{a}$, rather than given exogenously. In particular, $\bar{L}=\lambda_{e} /\left(\lambda_{e}+\delta_{e}\right)=\lambda_{b} /\left(\lambda_{b}+\delta_{b}\right)$, and (using $\left.\lambda_{e} / \delta_{e}=\lambda_{b} / \delta_{b}\right)$ it can easily be shown that $\bar{L}\left(\delta_{e}-\delta_{b}\right)=(1-\bar{L})\left(\lambda_{e}-\lambda_{b}\right)$. Thus the comparison between $V_{\ell}(b, \bar{L})-V_{\ell}(e, \bar{L})$ and $V_{f}(e, \bar{L})-V_{f}(e, \bar{L})$ simplifies to the comparison between $\pi_{\ell}$ and $\pi_{f}$, and the long-run policy is $e$ if $\pi_{\ell}<\pi_{f}$ and it is $b$ if $\pi_{\ell}>\pi_{f}$. In the context of the equation (5), here the extensive margin $L /(1-L)$ and a part of the intensive margin $\left(\delta_{e}-\delta_{b}\right) /\left(\lambda_{e}-\lambda_{b}\right)$ cancel out in the long run. Of course, in the short run, the current value of $L_{t}$ has an influence on the current policy choice.

One particular case that seems relevant in light of Olson's (1982) argument is the case where $\pi_{\ell}>\pi_{f}$. In this case, an economy which starts out as a "free society" with low $L$ and the free entry policy gradually builds up the special interest group of incumbents: $L$ grows over time. At some point, the special interest group grows sufficiently large and surpasses $L^{*}$ - after that, the economy stays in the blocking regime forever. Olson's analysis of postwar struggle in Great Britain ("British disease"), in contrast to the "miracles" in Germany and Japan, fits well with this type of dynamics. Olson writes, "with age British society has acquired so many strong organizations and collusions that it suffers from an institutional sclerosis that slows its adaptation to changing circumstances and technologies" (p.78). In fact, we can go further with our analysis - in the current case, the fundamental source of the convergence to the blocking regime is the fact that $\pi_{\ell}$ is larger than $\pi_{f}$. Therefore, one potential cure for this type of stagnation is to (permanently) raise the profitability of entrants in comparison to the incumbents.

Figure 2 is a numerical example of this case. The parameter values are: $\pi_{\ell}=3, \pi_{f}=1$, $\lambda_{e}=0.2, \delta_{e}=0.1, \lambda_{b}=0.1, \delta_{b}=0.05$, and $\beta=0.8{ }^{10}$ It draws the path of $L_{t}$ when it starts from $L_{1}=0.4$. The economy starts from a free entry regime, but as $L_{t}$ increases over

\footnotetext{
${ }^{10}$ The equilibrium is the "Regular Case" at all $t$.
} 


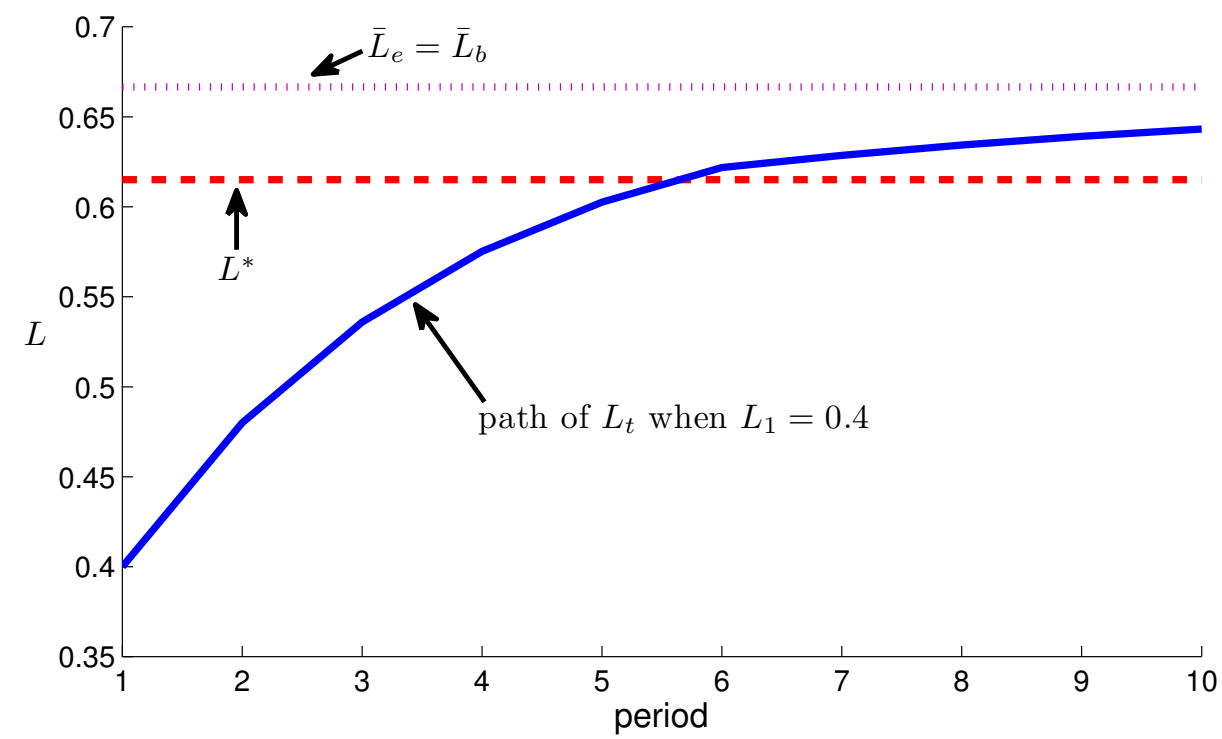

Figure 2: Example with "British disease" under common steady state

time, the incumbent coalition gains more political power. At period $6, L_{t}$ exceeds $L^{*}$ and the policy switches to entry blocking. $L_{t}$ continues to increase, and the policy is locked into entry blocking forever.

Figure 3 plots the path of the revenue flow for the policymaker that corresponds to the path of $L_{t}$ in Figure 2. In the static model, we have seen that the revenue of the policymaker is maximized when $L=L^{*}$. Similarly, Figure 3 shows that the revenue flow is high when $L_{t}$ is close to $L^{*}$.

\subsubsection{Political convergence II: Same profit}

Now consider the case where $\pi_{\ell}=\pi_{f}$ (call the common profit as $\pi$ ). As we will see below, in this situation it is also the case that $L^{*}$ is on the same side of $\bar{L}_{e}$ and $\bar{L}_{b}$ when $\bar{L}_{e} \neq \bar{L}_{b}$. First, note that the Bellman equation (17) is reduced to

$$
S(L)=\max _{a \in\{b, e\}}\{\mathcal{L}(a, L) \pi+\beta S(\mathcal{L}(a, L))\}
$$

With the standard argument (see Stokey, Lucas, and Prescott (1989)) it can be shown that $S(L)$ is strictly increasing. Therefore, at the right-hand side, a policy that maximizes $\mathcal{L}(a, L)$ 


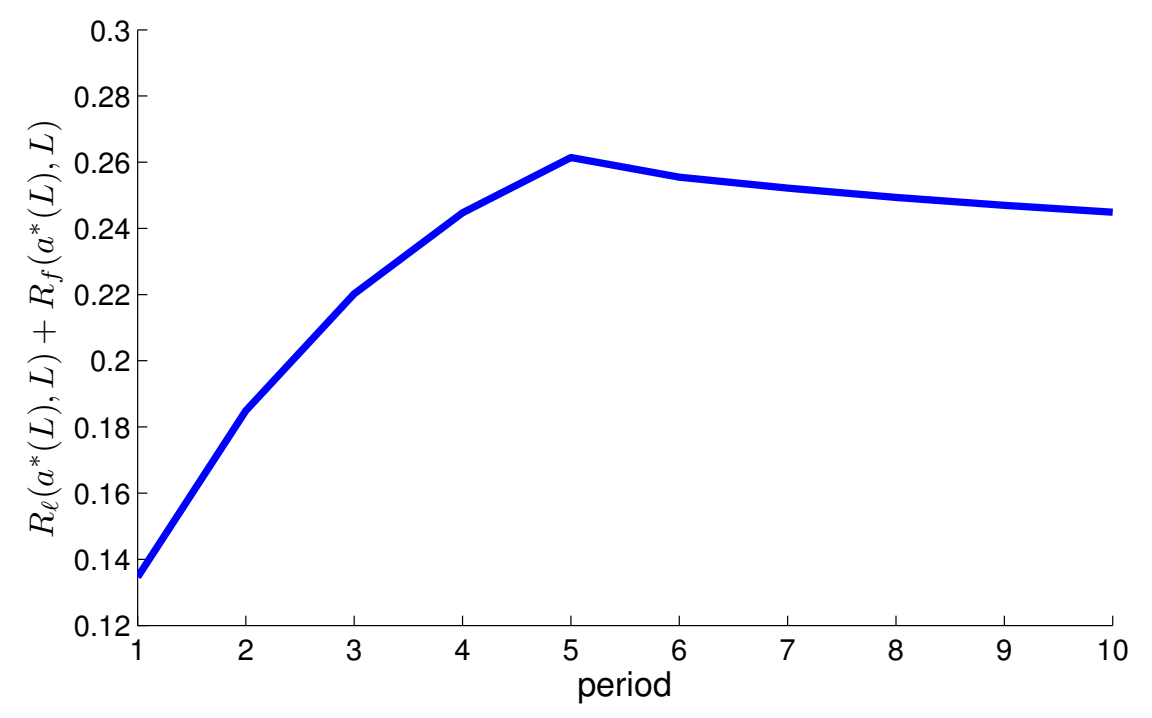

Figure 3: Example with "British disease" under common steady state: the revenue flow for the policymaker

is always chosen. The comparison between $\mathcal{L}(e, L)$ and $\mathcal{L}(b, L)$ leads us to the threshold rule for the policy:

$$
a= \begin{cases}e & \text { if } L<L^{*} \\ \{e, b\} & \text { if } L=L^{*} \\ b & \text { if } L>L^{*}\end{cases}
$$

where the threshold $L^{*}$ is

$$
L^{*}=\frac{\lambda_{e}-\lambda_{b}}{\left(\lambda_{e}-\lambda_{b}\right)+\left(\delta_{e}-\delta_{b}\right)} .
$$

Thus the threshold $L^{*}$ here is identical to the threshold in the static model (6) with $\pi_{\ell}=\pi_{f}$. It is straightforward to show that $\bar{L}_{b}>\bar{L}_{e}>L^{*}$ if $\bar{L}_{b}>\bar{L}_{e}$ (as in the first panel of Figure 1) and $L^{*}>\bar{L}_{e}>\bar{L}_{b}$ if $\bar{L}_{e}>\bar{L}_{b}$ (as in the fourth pane of Figure 1). Thus $L^{*}$ is always on the same side of $\bar{L}_{e}$ and $\bar{L}_{b}$ when $\bar{L}_{e} \neq \bar{L}_{b}$. (Clearly $L^{*}=\bar{L}_{e}=\bar{L}_{b}$ if $\bar{L}_{e}=\bar{L}_{b}$.)

In this case, the long-run behavior of the economy is determined by whether $\bar{L}_{e}$ is larger than $\bar{L}_{b}$. The long-run policy is $e$ if $\bar{L}_{e}>\bar{L}_{b}$ and it is $b$ if $\bar{L}_{b}>\bar{L}_{e}$. Therefore, the policy choice with free entry is likely to be sustained in an economy with a large value of $\bar{L}_{e}$, that is, either a large value of $\lambda_{e}$ or a small value of $\delta_{e}$.

As in the case of the common steady state, this case can also entail dynamics that resemble 


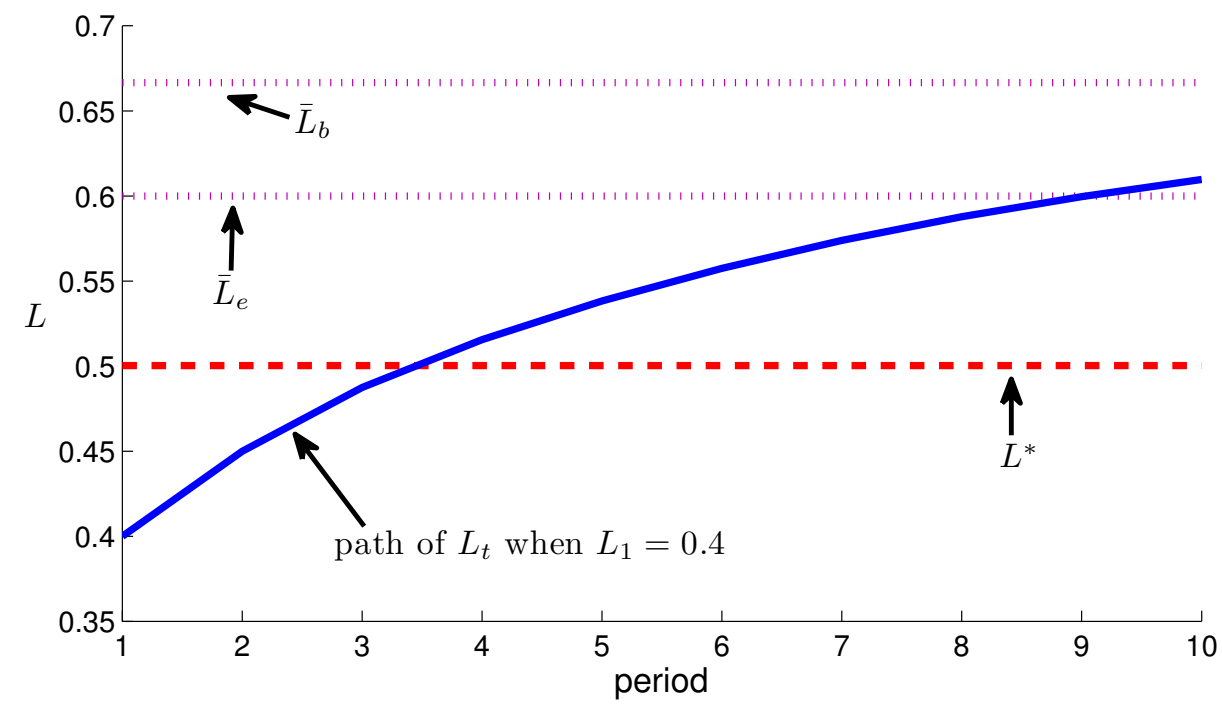

Figure 4: Example with "British disease" under common profit

"British disease" - the economy starts out from free entry and moves into (and gets stuck in) the blocking regime - this happens when $\bar{L}_{b}>\bar{L}_{e}$. Figure 4 is a numerical example of this case. The parameter values are: $\pi_{\ell}=1, \pi_{f}=1, \lambda_{e}=0.15, \delta_{e}=0.1, \lambda_{b}=0.1, \delta_{b}=0.05$, and $\beta=0.8{ }^{11}$ It draws the path of $L_{t}$ that starts from $L_{1}=0.4$. The economy starts from the free-entry regime. As time progresses, $L_{t}$ increases, and the political power of the incumbent coalition is strengthened. At period $4, L_{t}$ surpasses $L^{*}$, and the policy switches to entry blocking. $L_{t}$ converges to $L^{*}$, and the policy remains entry blocking forever. Figure 5 plots the corresponding revenue for the policymaker. As in the previous example, the revenue is high when $L_{t}$ is close to $L^{*}$.

In the current case, the fundamental cause for these dynamics is a relatively large value of $\bar{L}_{b}$. In this case, the cure for the "disease" should involve raising $\delta_{b}$ or $\lambda_{e}$, or reducing $\delta_{e}$ or $\lambda_{b}$.

\footnotetext{
${ }^{11}$ The equilibrium is the "Regular Case" at all $t$.
} 


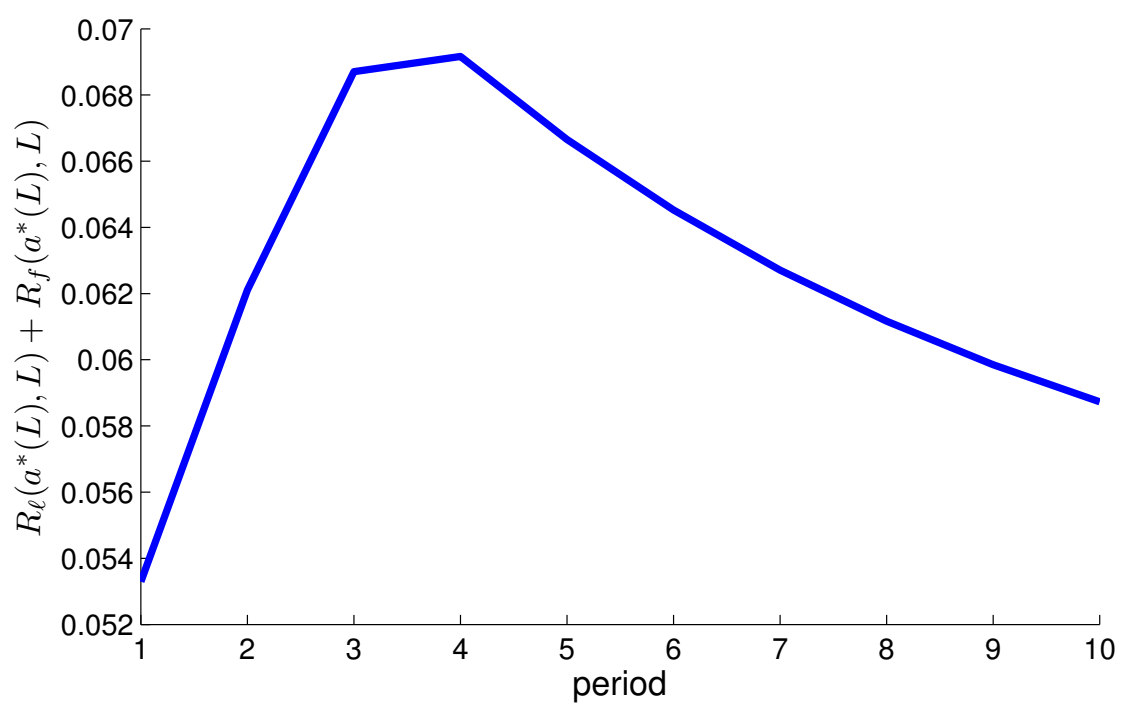

Figure 5: Example with "British disease" under common profit: the revenue flow for the policymaker

\subsubsection{Multiple steady states}

From the analysis of Section 3.4.1.2, we have seen that when $\pi_{\ell}=\pi_{f}$ and $\bar{L}_{b}>\bar{L}_{e}$, it is always the case that $\bar{L}_{b}>\bar{L}_{e}>L^{*}$, and the economy will eventually settle at the steady state with $L_{t}=\bar{L}_{b}$. As we increase $\pi_{f}$ relative to $\pi_{\ell}, L^{*}$ tends to increase and when $\pi_{f}$ is sufficiently larger than $\pi_{\ell}, L^{*}$ becomes larger than $\bar{L}_{e}$.

When $\bar{L}_{b}>L^{*}>\bar{L}_{e}$, the economy has multiple steady states. That is, depending on the the initial value of $L_{t}$ (call it $\left.L_{0}\right)$, the economy may end up in different steady states that have different political outcomes. Specifically, if $L_{0}<L^{*}$, the policy outcome is $a^{*}\left(L_{t}\right)=e$ for all $t$ and $L_{t}$ converges to $\bar{L}_{e}$. If $L_{0}>L^{*}$, the policy outcome is $a^{*}\left(L_{t}\right)=b$ for all $t$ and $L_{t}$ converges to $\bar{L}_{b}$. This case corresponds to the fifth panel of Figure 1.

Therefore, the initial condition matters in the long run, and some economies can get "stuck" in the policy that may not be desirable. This "history dependence" also implies that a temporary change in parameters, such as in $\pi_{\ell}, \pi_{f}, \lambda_{e}, \lambda_{b}, \sigma_{e}$, or $\sigma_{b}$, can have a permanent effect on the policy choice of the economy.

Figure 6 is a numerical example of the multiple steady states. The parameter values 


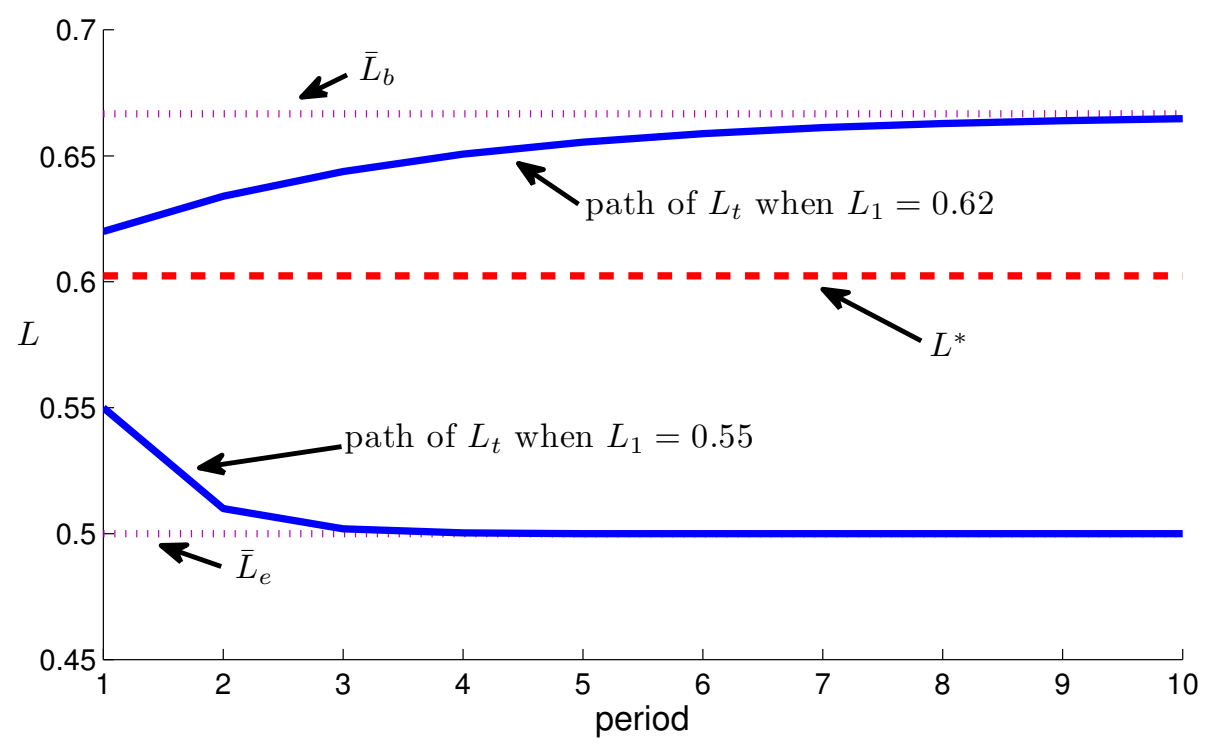

Figure 6: Example with multiple steady states

are: $\pi_{\ell}=1, \pi_{f}=2.2, \lambda_{e}=0.4, \delta_{e}=0.4, \lambda_{b}=0.2, \delta_{b}=0.1$, and $\beta=0.8$. The two solid lines are two sample paths. ${ }^{12}$ In the path above, $L_{1}=0.62$ and, since $L^{*}$ turns out to be 0.60 in this economy, the initial policy choice is $a^{*}\left(L_{1}\right)=b$. This raises $L_{t}$ and over time $L_{t}$ increases monotonically and approaches $\bar{L}_{b}$. The policy choice is $a^{*}\left(L_{t}\right)=b$ for all $t$. In the path below, $L_{1}=0.55$, which is below $L^{*}$, and $a^{*}\left(L_{t}\right)=e$ for all $t$. Figure 7 plots the corresponding paths of revenue for the policymaker. In both paths, revenue declines as $L_{t}$ moves farther from $L^{*}$.

The possibility of multiple steady state suggests one answer to a question we posed in the introduction: why do some countries institute heavy entry regulations while others do not? Even if the fundamental parameters are the same among countries, here history matters: if a country starts out with a large population (and therefore a large political power) of leaders who prefer entry blocking, a protective policy leads into more political power of the leaders in the future. If a country starts from relatively few leaders, a free environment for entry can persist.

\footnotetext{
${ }^{12}$ The equilibrium is the "Regular Case" for both paths at all $t$.
} 


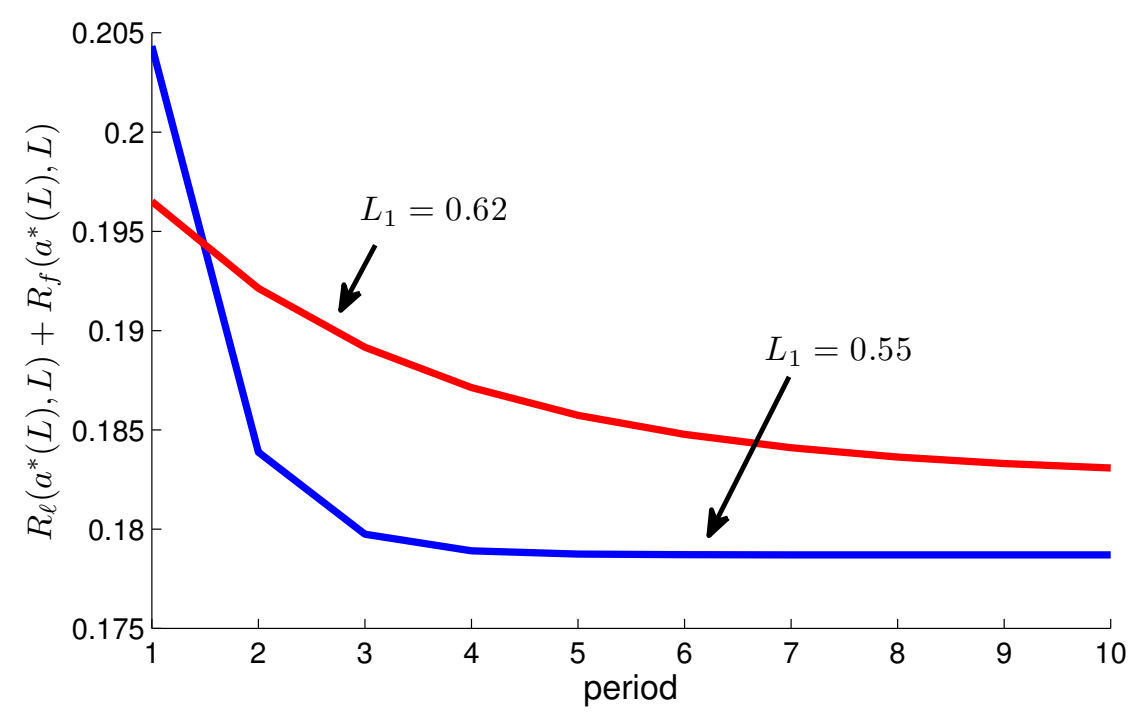

Figure 7: Example with multiple steady states: the revenue flow for the policymaker

When is it likely that multiple steady states exist in an economy? One key inequality is $\bar{L}_{b}>\bar{L}_{e}$, which is equivalent to $\lambda_{b} / \delta_{b}>\lambda_{e} / \delta_{e}$. This is the case when a blocking regime creates relatively more leaders (large $\lambda_{b}$ ) who tend to stay in the economy longer (small $\delta_{b}$ ). As in the case in Section 3.4.1.2, this creates feedback between the policy and the economy - an entry blocking policy increases the size of the leader coalition and strengthens their political power, leading to further lobbying towards blocking. If $\pi_{f}=\pi_{\ell}$, this leads to a political convergence to $\bar{L}_{b}$, as we have seen in Section 3.4.1.2. However, when $\pi_{f}>\pi_{\ell}$ (a relatively strong vintage effect), this profit difference counteracts the previous force and tends to move $L^{*}$ higher. When the difference between $\pi_{f}$ and $\pi_{\ell}$ is small, $\bar{L}_{e}>L^{*}$ and the long run steady state is $\bar{L}_{b}$, again as in Section 3.4.1.2. If the difference is very large, $L^{*} \geq \bar{L}_{b}$ and the long run steady state is $\bar{L}_{e}$. At an intermediate value of the difference, multiple steady states may arise. This is the situation where two conflicting forces have relatively balanced strengths. On one hand, the number of incumbents can expand quickly in the blocking regime. On the other, entrants have a strong profit motive to enter. When the initial value of $L_{t}$ is large, the first effect dominates, and when the initial value of $L_{t}$ is small, the second effect dominates. Once one effect dominates initially, that effect gains strength over time by the endogenous 
movement of $L_{t}$, and the economy gets stuck in that particular policy.

In this situation, the initial value of $L_{t}$ matters. An important policy implication is that a one-time (temporary) intervention that lowers $L_{t}$ can have the permanent effect of moving the economy from the entry blocking regime to the free entry regime. One way to accomplish this goal in a manner that is consistent with the constraints imposed by the political process is by increasing the profitability of entrants $\pi_{f}$ for certain amount of time. Proposition 9 formally shows this possibility.

Proposition 9 Suppose that $\bar{L}_{e}<L^{*}<\bar{L}_{b}$ and $L_{t}>L^{*}$. There exists some $T$ and $\pi_{f}^{\prime}>\pi_{f}$ such that if $\pi_{f}$ is increased to $\pi_{f}^{\prime}$ for $T$ periods from period $t$, then $a_{t^{\prime}}^{*}=e$ for all $t^{\prime} \geq t$.

\section{Proof. See Appendix B.}

As we have emphasized in Section 3.3.1, the "surplus-maximizing" nature of the equilibrium does not imply that the chosen policies are "optimal." In particular, since the consumers do not participate in the political process and the consumers are likely to benefit from the free entry regime, it is possible that the political outcome will end up "getting stuck" in the entryblocking regime even when the free entry policy is the desirable one from the entire economy's welfare standpoint. Since neither the incumbent coalition nor the potential entrant coalition internalizes the consumer surplus, there is a role for some outside intervention in our model. Proposition 9 shows that, if this situation arises in this multiple steady-state environment, an intervention that raises $\pi_{f}$ temporarily can pull out the economy out of the entry-blocking regime and move it to the free-entry regime permanently.

\subsubsection{Cyclical dynamics}

Finally, our framework also permits a cyclical dynamics. Many previous studies, such as Krusell and Ríos-Rull (1996), Bridgman et al (2004), and Bellettini and Ottaviano (2005), highlighted the ability of their models to generate policy cycles.

Cyclical dynamics occur when $\bar{L}_{b}<L^{*}<\bar{L}_{e}$. This corresponds to the last panel of Figure 1. This occurs when $\lambda_{b} / \delta_{b}<\lambda_{e} / \delta_{e}$ and $\pi_{\ell}$ is sufficiently larger than $\pi_{f}$ (but not too large). When the economy starts from the free entry regime, $L_{t}$ increases, and this strengthens the 


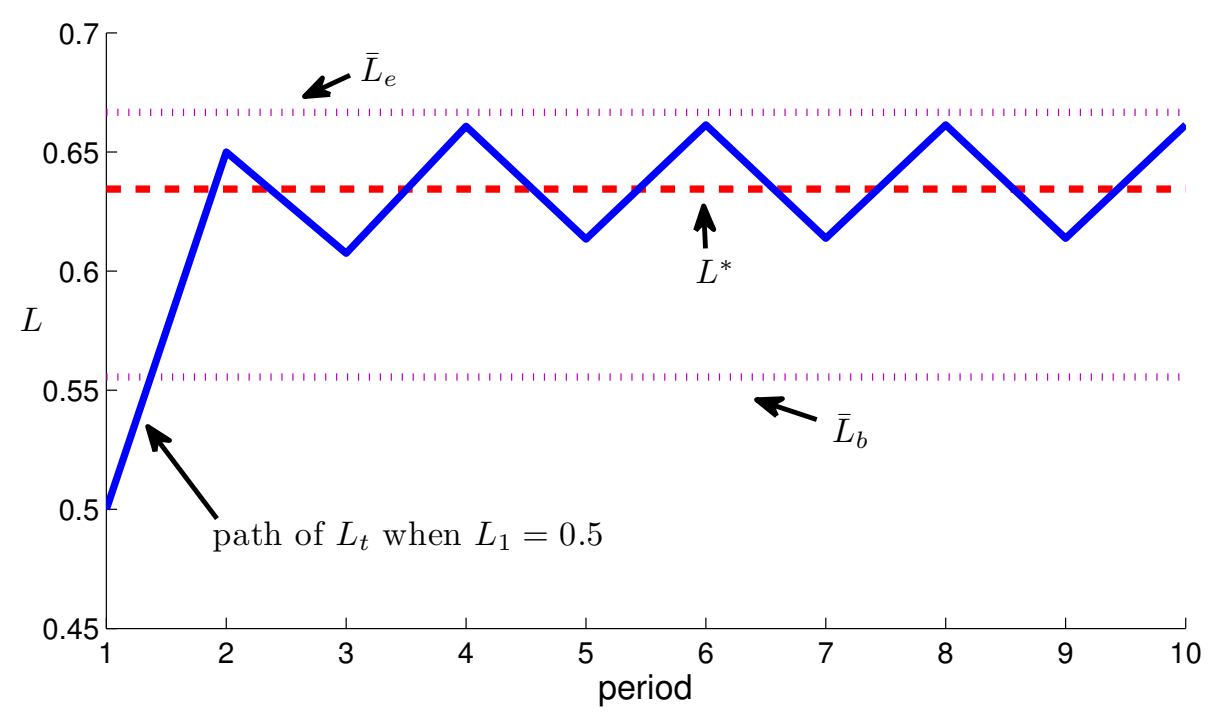

Figure 8: Example with cyclical dynamics

incumbents' coalition and the entry blocking policy follows. The blocking regime leads to a small $L_{t}$, weakens the incumbents' political power, and it leads to the free entry regime. This interaction repeats, exhibiting cyclical dynamics in $L_{t}$ and frequent policy switches.

Figure 8 is an example of cyclical dynamics. The parameter values are: $\pi_{\ell}=7, \pi_{f}=1$, $\lambda_{e}=0.6, \delta_{e}=0.3, \lambda_{b}=0.25, \delta_{b}=0.2$, and $\beta=0.8 .{ }^{13}$ Figure 9 is the revenue path for the policymaker. As in the previous cases, the revenue is high when $L_{t}$ is close to $L^{*}$.

\section{Conclusion}

Why does an inefficient policy persist? In particular, why are there many governments that impose such high barriers for the entry of new firms? We consider this question by constructing a simple model of political economy. Our model can generate dynamics where the interaction of the economy and the endogenous policy can lead to a situation where the policymaker chooses a policy with a high entry cost for a long time. A particularly important case is where the economy can exhibit multiple steady states. In that case, economies with the same fundamentals (same parameter values) can end up choosing very different policies

\footnotetext{
${ }^{13}$ The equilibrium is the "Regular Case" at all $t$.
} 


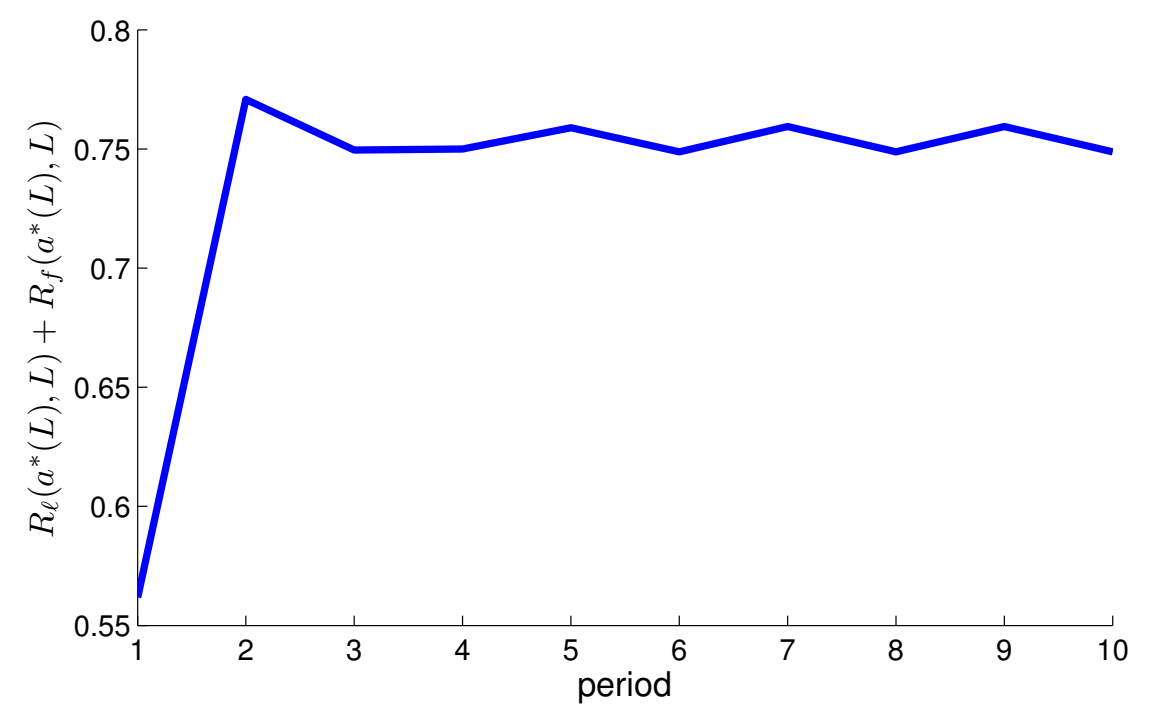

Figure 9: Example with cyclical dynamics: the revenue flow for the policymaker

in the long run if they start from two different initial situations. In a situation like this, a temporary policy can have a permanent effect on the policy and the economic performance.

In addition to multiple steady states, our model permits very rich possibilities of the dynamics of the economy. We described the dynamics that resemble a "British disease," where an economy that starts out with an environment that fosters free entry may develop special interest groups in the long run and end up blocking entry of new firms. We also highlighted a situation where political cycles over time may arise.

We have shown that the equilibrium policy maximizes the (present-value of) total surplus. This does not mean that the policy chosen is the "optimal" one. It is important to emphasize that the "total surplus" here includes only the surpluses of the participants in the political process. In our model, the firms and the policymaker participate in the policy decision, but the consumers do not participate. The consumer's welfare is ignored in the "total surplus." Hayek (1979), referring to Olson (1965), writes, "it is impossible in principle to organize all interests ... in consequence the organization of certain large groups assisted by government leads to a persistent exploitation of unorganized and unorganizable groups" (p. 97). The consumer here is one example of "unorganized and unorganizable groups" that can suffer from 
the political influences of the organized special interest groups (in particular, the firms that prefer "blocking"). As we discussed in the introduction, it is likely that the consumers benefit from entry of new firms. The economic growth literature based on "creative destruction" has emphasized the importance of new firm entry in the context of economic growth and development. Incorporating consumers' welfare explicitly and analyzing the political economy of entry and exit in a model with an explicit growth process are important future research topics. ${ }^{14}$ With these models, we will be able to further analyze the normative consequences of these political economy outcomes.

The result that an economy can get trapped into an "inefficient steady state," as in the case of "British disease" or in the case with multiple steady states, calls for appropriate interventions from outside. As Acemoglu and Robinson (2012) write, in these situations "the presence of markets is not by itself a guarantee of inclusive institutions. Markets, left to their own devices, can cease to be inclusive, becoming increasingly dominated by the economically and politically powerful. Inclusive economic institutions require not just markets, but inclusive markets that create a level playing field and economic opportunities for majority of the people" (p. 323). In order to ensure that the inclusive markets ("free entry" in our terminology) persist, we may have to appeal to interventions that influence the distribution of political power (the value $L_{t}$ in our model) or the incentive of entry and exit (the values of $\lambda \mathrm{s}, \delta \mathrm{s}$, and $\pi \mathrm{s}$ ). In some situations the intervention can be temporary (in the case with multiple steady states) and in other situations it may have to be permanent (in the case of "British disease").

Another important extension of our analysis is incorporating exogenous "aggregate shocks" into the model economy. In an environment where multiple steady states (more precisely, multiple ergodic distributions) exist, a temporary aggregate shock can move the economy into a situation that leads to a different long-run outcome. ${ }^{15}$ There, history matters not only in the sense that the initial state matters, but also in the sense that the entire realizations of

\footnotetext{
${ }^{14}$ The model in Appendix $\mathrm{A}$ is one such an attempt.

${ }^{15}$ Acemoglu and Robinson (2012) use the term "critical juncture" in referring to such events that "disrupt the existing political and balances in one or many societies" (p. 431). Their examples include the Black Death, the opening of Atlantic trade routes, and the Industrial Revolution.
} 
the aggregate shocks matter for the long-run outcome (ergodic distribution) of the economy. 


\section{Appendix}

\section{A An endogenous growth model}

This section embeds a "quality ladder" style endogenous growth model into our dynamic political economy model in the main text. The model is considerably more complex than the one in the main text. We obtain a similar result to the original model. In particular, we present an example where multiple balanced growth paths exist. One benefit of the formulation here is that we can explicitly introduce the consumers who are not in organized coalitions, and analyze the welfare properties of the model.

\section{A.1 Model setup}

There are three types of agents in the economy: entrepreneurs $(e)$, workers $(w)$, and a policymaker $(p)$. The population of the entrepreneurs is 1 and the population of the workers is $N$. All agents maximize the present value of their expected consumption:

$$
E_{0}\left[\sum_{t=0}^{\infty} \beta^{t} c_{t}^{j}\right],
$$

where $\beta \in(0,1)$ and $j \in\{e, w, p\}$. There are continuum of industries on $[0,1]$ that produces differentiated intermediate goods, and each industry corresponds to an entrepreneur (that is, entrepreneur $i$ produces in industry $i$ ). Each worker supplies one unit of labor inelastically for the production of the final (consumption) good. The final (consumption) good is produced by the production function

$$
O_{t}=N^{1-\alpha} \int_{0}^{1} A_{i t}^{1-\alpha} x_{i t}^{\alpha} d i
$$

where $\alpha \in(0,1), A_{i t}>0$ is the "quality" of the intermediate good from industry $i$, and $x_{i t}$ is the quantity of the intermediate good $i$ that is used for the production. The final good industry is perfectly competitive, and pays the marginal product of labor to the workers and and the marginal product of the intermediate input to the intermediate good producer.

At a point in time, each intermediate good industry is either "monopolistic" or "com-

petitive." When monopolistic, the intermediate good of that industry is solely produced by 
the industry leader who invented the cutting-edge quality of good $i$. (One interpretation is that when the cutting-edge quality is invented, the government provides the entrepreneur a monopoly right by, for example, granting a patent protection.) When the cutting-edge quality is imitated (patent expires), the industry become competitive, and the good $i$ is produced by the "competitive fringe" which acts as a price-taker.

An monopolistic industry at the start of time $t$ becomes competitive with probability $\delta_{a}$. (As in the main text, $\delta_{a}$ depends on the policy regime a.) If it becomes competitive, the competitive fringe produces, and the entrepreneur is forced out of the market (and becomes a "potential entrant") until it can innovate the next quality. If it remains monopolistic (with probability $\left.\left(1-\delta_{a}\right)\right)$, the entrepreneur produces the intermediate input competitively. The production of one unit of intermediate good requires one unit of final good. Since the price of the intermediate good is its marginal product in final good production, the maximization problem for the monopolist is:

$$
\max _{x_{i t}}\left(\alpha N^{1-\alpha} A_{i t}^{1-\alpha} x_{i t}^{\alpha-1}-1\right) x_{i t}
$$

The solution is

$$
x_{i t}=\alpha^{\frac{2}{1-\alpha}} A_{i t} N
$$

and the profit is

$$
\pi_{i t}=\alpha^{\frac{1+\alpha}{1-\alpha}}(1-\alpha) A_{i t} N
$$

In the competitive industry, the price is equal to the marginal cost, therefore

$$
x_{i t}=\alpha^{\frac{1}{1-\alpha}} A_{i t} N
$$

For simplicity, we set $N=1$ in the following. Let the number of monopolistic industries at the beginning of period $t$ be $L_{t}$. Then, at period $t, \delta_{a} L_{t}$ number of industries among them operate as competitive industries and $\left(1-\delta_{a}\right) L_{t}$ number of industries among them operate as monopolistic.

If an industry start as a competitive industry, there is a probability $\lambda_{a}$ that the potential entrant succeeds in innovating the next generation of quality and becomes a monopolist. 
Suppose that the new quality is $\gamma>1$ times more productive than the previous quality (that is, $\gamma A_{i, t-1}$ is the "true" productivity if this intermediate good is used). Here, we allow the "actual" productivity at the period of the innovation can be larger or smaller than the true productivity: $\theta \gamma A_{i, t-1}$. If $\theta$ is less than one, the first period "actual" productivity is lower than the "true" productivity, possibly because of the necessity of learning for the new technology. If $\theta$ is larger than one, then the actual productivity is higher at the initial period than the true productivity, possibly because the new vintage of technology provides a temporary boost to the productivity.

Since $\lambda_{a}\left(1-L_{t}\right)$ industries innovate and $\left(1-\lambda_{a}\right)\left(1-L_{t}\right)$ industries remain competitive in this part of the economy, the average productivity for the whole economy at the end of period $t$ is

$$
\bar{A}_{t} \equiv \int_{0}^{1} A_{i t} d i=\lambda_{a}\left(1-L_{t}\right) \gamma \bar{A}_{t-1}^{c}+\left(1-\lambda_{a}\right)\left(1-L_{t}\right) \bar{A}_{t-1}^{c}+L_{t} \bar{A}_{t-1}^{m},
$$

where $\bar{A}_{t-1}^{c}$ is the average productivity of the competitive industries and $\bar{A}_{t-1}^{m}$ is the average productivity of the monopolistic industries. The averages evolve according to:

$$
\bar{A}_{t}^{m}=\frac{\lambda_{a}\left(1-L_{t}\right) \gamma \bar{A}_{t-1}^{c}+\left(1-\delta_{a}\right) L_{t} \bar{A}_{t-1}^{m}}{\lambda_{a}\left(1-L_{t}\right)+\left(1-\delta_{a}\right) L_{t}}
$$

and

$$
\bar{A}_{t}^{c}=\frac{\left(1-\lambda_{a}\right)\left(1-L_{t}\right) \bar{A}_{t-1}^{c}+\delta_{a} L_{t} \bar{A}_{t-1}^{m}}{\left(1-\lambda_{a}\right)\left(1-L_{t}\right)+\delta_{a} L_{t}} .
$$

In order to facilitate the mapping to the main text, we make a simplifying assumption.

Assumption 1 We assume that at the end of the period, the productivity of all industries equalize to the average productivity. That is,

$$
A_{i t}=\bar{A}_{t}=\bar{A}_{t}^{c}=\bar{A}_{t}^{m}
$$

for all $i$ and $t$, at the end of period $t$. The industry structure (monopolistic or competitive) remains the same.

Thus, at the end of period $t$,

$$
\bar{A}_{t}=\bar{A}_{t-1}+\bar{A}_{t-1} \lambda_{a}\left(1-L_{t}\right)(\gamma-1)
$$


and the gross growth rate of the technology is

$$
\frac{\bar{A}_{t}}{\bar{A}_{t-1}}=g_{a}\left(L_{t}\right) \equiv \lambda_{a}\left(1-L_{t}\right)(\gamma-1)+1
$$

At the individual industry level, $A_{i t}=\bar{A}_{t-1}$ if the industry does not innovate at time $t$ and $A_{i t}=\theta \gamma \bar{A}_{t-1}$ if it innovates. Clearly, Assumption 1 is extreme and unrealistic. We can still define and compute the Truthful Markov Perfect Equilibrium without this assumption, but the surplus-maximizing property (Proposition 4 in the main text) does not hold, and therefore the characterization is much more complex.

\section{A.2 Constrained social optimum}

In this section, we introduce a utilitarian social welfare problem. In a subsequent section, we will contrast it with the political-economy equilibrium.

The hypothetical social planner, who makes the political decision, is utilitarian. She makes the policy decision based on the total welfare of the economy, and is not swayed by the political process. We assume that she gives equal weight to the utility of all participants in the economy. Since the utility functions are all linear, the social welfare function is simply discounted net output and is given by:

$$
\sum_{t=0}^{\infty} \beta^{t}\left[\int_{0}^{1} A_{i t}^{1-\alpha} x_{i t}^{\alpha} d i-\int_{0}^{1} x_{i t} d i\right] .
$$

We assume that the fundamental parameter values (which reflect the patent system and the system for financing innovation) and the market structure (monopoly/competitive) cannot be altered by the social planner. (Thus, the resulting outcome is a "constrained social optimum.") The only choice variable in the social planning problem is the choice of the entry regimes (between free entry and entry blocking).

Assumption 1 ensures that the state variables in this problem are average productivity $\bar{A}_{t-1}$ and the mass of monopolistic industries $L_{t}$.

The contribution to net output of a monopolistic firm with productivity $A_{i t}$ is

$$
A_{i t}^{1-\alpha} x_{i t}^{\alpha}-x_{i t}=A_{i t}\left[\alpha^{\frac{2 \alpha}{1-\alpha}}-\alpha^{\frac{2}{1-\alpha}}\right] .
$$


Similarly, for a competitive industry,

$$
A_{i t}^{1-\alpha} x_{i t}^{\alpha}-x_{i t}=A_{i t}\left[\alpha^{\frac{\alpha}{1-\alpha}}-\alpha^{\frac{1}{1-\alpha}}\right] .
$$

Then the current period, output for given $L_{t}, \bar{A}_{t-1}$ and $a$ is:

$$
\left(\delta_{a} L_{t}+\left(1-\lambda_{a}\right)\left(1-L_{t}\right)\right) z_{c} \bar{A}_{t-1}+\left(\left(1-\delta_{a}\right) L_{t}+\lambda_{a}\left(1-L_{t}\right) \theta \gamma\right) z_{m} \bar{A}_{t-1},
$$

where $z_{c} \equiv \alpha^{\frac{\alpha}{1-\alpha}}-\alpha^{\frac{1}{1-\alpha}}$ and $z_{m} \equiv \alpha^{\frac{2 \alpha}{1-\alpha}}-\alpha^{\frac{2}{1-\alpha}}$. Note that $z_{m}=\alpha^{\frac{\alpha}{1-\alpha}}(1+\alpha) z_{c}$.

Denote $L=L_{t}$ and $\bar{A}=\bar{A}_{t-1}$. The problem for the social planner is given by the following Bellman equation:

$W(L, \bar{A})=\max _{a \in\{e, b\}}\left(\delta_{a} L+\left(1-\lambda_{a}\right)(1-L)\right) z_{c} \bar{A}+\left(\left(1-\delta_{a}\right) L+\lambda_{a}(1-L) \theta \gamma\right) z_{m} \bar{A}+\beta W\left(\mathcal{L}(a, L), g_{a}(L) \bar{A}\right)$.

It is easy to see that $W(L, \bar{A})=\bar{A} W(L, 1)$ for all $\bar{A}, L$. So if we denote $\tilde{W}(L)=W(L, 1)$, the equation above can be rewritten more simply as:

$$
\tilde{W}(L)=\max _{a \in\{e, b\}}\left(\delta_{a} L+\left(1-\lambda_{a}\right)(1-L)\right) z_{c}+\left(\left(1-\delta_{a}\right) L+\lambda_{a}(1-L) \theta \gamma\right) z_{m}+\beta g_{a}(L) \tilde{W}(\mathcal{L}(a, L))
$$

We make the following assumption:

\section{Assumption 2}

$$
\max _{a \in\{e, b\}, L \in[0,1]} g_{a}(L)<\frac{1}{\beta} .
$$

This implies that the Bellman operator defined above is a contraction mapping with a bounded and continuous fixed point.

\section{A.3 Political equilibrium}

Here we introduce the political economy model in this environment. As before, we will concentrate on symmetric Markov equilibria, but now one of the relevant state variables is the average productivity $\bar{A}$. An old monopolist makes a profit of $\alpha^{\frac{1+\alpha}{1-\alpha}}(1-\alpha) \bar{A}$, whereas a new monopolist makes a profit of $\alpha^{\frac{1+\alpha}{1-\alpha}}(1-\alpha) \theta \gamma \bar{A}$. Let $\kappa \equiv \alpha^{\frac{1+\alpha}{1-\alpha}}(1-\alpha)$.

$n_{i}(L, \bar{A})$ and $v_{i}(a, L, \bar{A})$ are defined similarly as in the main body of the paper. They satisfy the following equations:

$$
v_{\ell}(a, L, \bar{A})=\left(1-\delta_{a}\right)\left(\kappa \bar{A}+\beta n_{\ell}\left(\mathcal{L}(a, L), g_{a}(L) \bar{A}\right)\right)+\delta_{a} \beta n_{f}\left(\mathcal{L}(a, L), g_{a}(L) \bar{A}\right)
$$


and

$$
v_{f}(a, L, \bar{A})=\lambda_{a}\left(\kappa \theta \gamma \bar{A}+\beta n_{\ell}\left(\mathcal{L}(a, L), g_{a}(L) \bar{A}\right)\right)+\left(1-\lambda_{a}\right) \beta n_{f}\left(\mathcal{L}(a, L), g_{a}(L) \bar{A}\right)
$$

The total gross values of the coalitions are simply $V_{\ell}(a, L, \bar{A})=L v_{\ell}(a, L, \bar{A})$ and $V_{f}(a, L, \bar{A})=$ $(1-L) v_{f}(a, L, \bar{A})$.

The menu of payment $R$, the equilibrium net value of a coalition $n$ and the government's value $G$ are defined similarly as in the main text; a truthful strategy and the Truthful Markov Perfect Equilibrium are also similarly defined.

We conjecture that $v_{i}(a, L, \bar{A})=\bar{A} v_{i}(a, L, 1)$ and $n_{i}(L, \bar{A})=\bar{A} n_{i}(L, 1)$. Denoting $\tilde{v}_{i}(a, L) \equiv$ $v_{i}(a, L, 1)$ and $\tilde{n}_{i}(L) \equiv n_{i}(L, 1)$, we have:

$$
\begin{gathered}
\tilde{v}_{\ell}(a, L)=\left(1-\delta_{a}\right)\left(\kappa+\beta g_{a}(L) \tilde{n}_{\ell}(\mathcal{L}(a, L))\right)+\delta_{a} \beta g_{a}(L) \tilde{n}_{f}(\mathcal{L}(a, L)), \\
\tilde{v}_{f}(a, L)=\lambda_{a}\left(\kappa \theta \gamma+\beta g_{a}(L) \tilde{n}_{\ell}(\mathcal{L}(a, L))\right)+\left(1-\lambda_{a}\right) \beta g_{a}(L) \tilde{n}_{f}(\mathcal{L}(a, L)) .
\end{gathered}
$$

From the definition of $n$ and above, $\tilde{v}$ and $\tilde{n}$ do not depend on $\bar{A}$, and thus the conjecture is verified. Following a similar argument as in the main body of the paper, we can establish the existence of discrete approximation of the equilibrium.

We define the total surplus as $S(L, \bar{A}) \equiv L n_{\ell}(L, \bar{A})+(1-L) n_{f}(L, \bar{A})+G(L, \bar{A})$. In equilibrium, it satisfies the equation (the proof is similar to the one for Proposition 4):

$$
S(L, \bar{A})=\max _{a \in\{e, b\}}\left(1-\delta_{a}\right) L \kappa \bar{A}+\lambda_{a}(1-L) \kappa \theta \gamma \bar{A}+\beta S\left(\mathcal{L}(a, L), \bar{A} g_{a}(L)\right) .
$$

By a standard argument, we can establish that the Bellman equation above is a contraction mapping and $S(L, \bar{A})=\bar{A} S(L, 1)$. Thus again, by defining $\tilde{S}(L) \equiv S(L, 1)$, we have

$$
\tilde{S}(L)=\max _{a \in\{e, b\}}\left(1-\delta_{a}\right) L \kappa+\lambda_{a}(1-L) \kappa \theta \gamma+\beta g_{a}(L) \tilde{S}(\mathcal{L}(a, L))
$$

\section{A.4 The growth rate and the individual welfare in the steady state}

In the following, we focus on a steady state (balanced growth path). As in the main text, there are parametric configurations where the Truthful Markov Perfect Equilibrium does not involve a steady state - we rule out such parametric configurations in the analysis below. ${ }^{16}$

\footnotetext{
${ }^{16}$ The current framework can handle these situations, but here we ignore this issue for simplicity.
} 
The steady state value of $L_{t}$, if the same policy regime continues forever, is $\bar{L}=\lambda_{a} /\left(\lambda_{a}+\right.$ $\left.\delta_{a}\right)$. The steady-state growth rate of $\bar{A}_{t}$ is

$$
\frac{\bar{A}_{t}}{\bar{A}_{t-1}}=\bar{g}_{a} \equiv(\gamma-1) \frac{\lambda_{a} \delta_{a}}{\lambda_{a}+\delta_{a}}+1
$$

It is straightforward to see that $\bar{g}_{a}$ is increasing in $\lambda_{a}$ and $\delta_{a}$. Given our assumption that $\lambda_{e}>\lambda_{b}$ and $\delta_{e}>\delta_{b}, \bar{g}_{a}$ is higher with the free entry regime.

As in the previous section, let the present discounted value of a "leader" entrepreneur (who entered the market at time $t$ as a monopolist) as $v_{\ell t}$, and let $\tilde{v}_{\ell t} \equiv v_{\ell t} / \bar{A}_{t-1}$. In the steady state, $\tilde{v}_{\ell t}$ is constant, and thus we denote it as $\tilde{v}_{\ell}$. Similarly, we denote the present discounted value of a "follower" entrepreneur (who entered a market at time $t$ as a potential entrant) be $v_{f t}$ and define $\tilde{v}_{f t} \equiv v_{f t} / \bar{A}_{t-1}$. Again, $\tilde{v}_{f t}$ is constant in the steady state and we denote it $\tilde{v}_{f}$. Let $\pi_{\ell t}$ be the profit in (A.1) and $\tilde{\pi}_{\ell} \equiv \pi_{\ell t} / \bar{A}_{t-1}\left(\tilde{\pi}_{\ell}\right.$ is always constant). Similarly, let $\pi_{f t}$ be the profit in (A.1) and $\tilde{\pi}_{f} \equiv \pi_{f t} / \bar{A}_{t}$. Note that $\tilde{\pi}_{f}=\theta \gamma \tilde{\pi}_{\ell}$, and thus $\tilde{\pi}_{f}$ can be larger or smaller than $\tilde{\pi}_{\ell}$.

Similarly to the main text, let $\tilde{n}_{\ell}$ and $\tilde{n}_{f}$ be the net payoffs of a leader and a follower respectively, normalized by $\bar{A}_{t-1}$ as above, after making the payment to the policymaker. That is, denoting $\tilde{r}_{\ell}$ and $\tilde{r}_{f}$ to be the normalized per-entrepreneur payment to the policymaker,

$$
\tilde{n}_{\ell}=\tilde{v}_{\ell}-\tilde{r}_{\ell}
$$

and

$$
\tilde{n}_{f}=\tilde{v}_{f}-\tilde{r}_{f}
$$

hold. Then,

$$
\tilde{v}_{\ell}=\left(1-\delta_{a}\right)\left(\pi_{\ell}+\beta \bar{g}_{a} \tilde{n}_{\ell}\right)+\delta_{a} \beta \bar{g}_{a} \tilde{n}_{f}
$$

and

$$
\tilde{v}_{f}=\lambda_{a}\left(\pi_{f}+\beta \bar{g}_{a} \tilde{n}_{\ell}\right)+\left(1-\lambda_{a}\right) \beta \bar{g}_{a} \tilde{n}_{f}
$$

determine the values of $\tilde{v}_{\ell}$ and $\tilde{v}_{f}$. Of course, $a, \tilde{r}_{\ell}$, and $\tilde{r}_{f}$ are equilibrium objects here, and when the Truthful Markov Perfect Equilibrium is defined in this economy (as in the main 
text), we have to evaluate the off the equilibrium payoffs (which involves off the steady state behavior).

The utility for the policymaker is (again, for given $a, \tilde{r}_{\ell}$, and $\tilde{r}_{f}$ and after normalizing)

$$
\tilde{G}=\tilde{r}_{\ell} \bar{L}_{a}+\tilde{r}_{f}\left(1-\bar{L}_{a}\right)+\beta \bar{g}_{a} \tilde{G}
$$

Above Bellman equations are very similar to the main text. The only difference is that the "effective discount factor" $\beta \bar{g}_{a}$ is policy variant. (It also depends on $L_{t}$ outside the steady state.)

In order to calculate the utility for the workers, one need to compute the income (which is equal to consumption with the linear utility) for the workers. The workers are paid their marginal product. The marginal product in equilibrium is $(1-\alpha) \int_{0}^{1} A_{i t}{ }^{1-\alpha} x_{i t}^{\alpha} d i$. Let $X_{i t}=$ $A_{i t}{ }^{1-\alpha} x_{i t}^{\alpha}$ be the quality adjusted contribution of intermediate good $i$. With Assumption 1 , this is $X_{i t}=\alpha^{\frac{2 \alpha}{1-\alpha}} \theta \gamma \bar{A}_{t-1}$ in an industry that innovates, and $\alpha^{\frac{2 \alpha}{1-\alpha}} \bar{A}_{t-1}$ in an industry that is monopolistic from the previous period. In a competitive industry, it is $\alpha^{\frac{\alpha}{1-\alpha}} \bar{A}_{t-1}$. Therefore,

$$
c_{i}^{w}=\frac{\theta \gamma \lambda_{a} \delta_{a} \alpha^{\frac{2 \alpha}{1-\alpha}}+\left(1-\lambda_{a}\right) \delta_{a} \alpha^{\frac{\alpha}{1-\alpha}}+\lambda_{a}\left(1-\delta_{a}\right) \alpha^{\frac{2 \alpha}{1-\alpha}}+\lambda_{a} \delta_{a} \alpha^{\frac{\alpha}{1-\alpha}}}{\lambda_{a}+\delta_{a}}(1-\alpha) \bar{A}_{t-1}
$$

is the income per worker in the steady state. Thus the flow benefit for the worker is proportional to

$$
\frac{\theta \gamma \lambda_{a} \delta_{a} \alpha^{\frac{\alpha}{1-\alpha}}+\left(1-\lambda_{a}\right) \delta_{a}+\lambda_{a}\left(1-\delta_{a}\right) \alpha^{\frac{\alpha}{1-\alpha}}+\lambda_{a} \delta_{a}}{\lambda_{a}+\delta_{a}} \bar{A}_{t-1}
$$

while the total flow surplus of the entrepreneurs and the policymaker (combined) is proportional to (we can see this by summing up the values for the entrepreneurs and the policymaker above, or directly from (A.3))

$$
\frac{\theta \gamma \lambda_{a} \delta_{a}+\lambda_{a}\left(1-\delta_{a}\right)}{\lambda_{a}+\delta_{a}} \bar{A}_{t-1}
$$

The social planner in Section A.2 looks at the entire social welfare (the combination of the above two) and it is proportional to (from (A.2))

$$
\frac{\theta \gamma \lambda_{a} \delta_{a} \alpha^{\frac{\alpha}{1-\alpha}}(1+\alpha)+\left(1-\lambda_{a}\right) \delta_{a}+\lambda_{a}\left(1-\delta_{a}\right) \alpha^{\frac{\alpha}{1-\alpha}}(1+\alpha)+\lambda_{a} \delta_{a}}{\lambda_{a}+\delta_{a}} \bar{A}_{t-1} .
$$




\begin{tabular}{|c|c|c|c|c|c|c|c|}
\hline$\lambda_{e}$ & $\delta_{e}$ & $\lambda_{b}$ & $\delta_{b}$ & $\gamma$ & $\beta$ & $\theta$ & $\alpha$ \\
\hline 0.70 & 0.50 & 0.25 & 0.10 & 1.10 & 0.80 & 1.00 & 0.30 \\
\hline
\end{tabular}

Table 1: Parameter values

\begin{tabular}{|l||c|c|c|}
\hline & $w$ & $e$ and $p$ & total \\
\hline $\bar{L}_{e}$ & 1.85 & 0.26 & 2.11 \\
\hline $\bar{L}_{b}$ & 1.54 & 0.28 & 1.82 \\
\hline
\end{tabular}

Table 2: Steady-state welfare, starting from $\bar{A}_{0}=1$

The discrepancy comes from that the entrepreneurs capture the profit only in monopolistic industries, while the workers can receive benefit from both monopolistic and competitive industries (even more from the competitive industries). This discrepancy can lead to the policy choice that are beneficial to the entrepreneurs and the policymaker but not for the workers (and the whole economy).

\section{A.5 A numerical example of multiple steady states}

In this section, we provide a numerical example where there are multiple steady states in the political equilibrium.

The parameter values are shown in Table 1 . The steady state values of $L$ are $\bar{L}_{e}=0.58$ and $\bar{L}_{b}=0.71$. The steady-state (net) growth rate is $\bar{g}_{e}-1=2.9 \%$ under the free entry regime and $\bar{g}_{b}-1=0.7 \%$ under the entry blocking regime.

The social planner solves the problem (A.2) and the optimal solution is $a=e$ for all $L$. The political equilibrium is the solution to the problem (A.3) and the solution has the threshold property as in the main text. The threshold $L^{*}=0.61$. Thus, as in Section 3.4.2 (also see Figure 6), the political equilibrium exhibits the multiple steady states. If $L_{1}>L^{*}$, the economy converges to the blocking regime with low growth, and if $L_{1}<L^{*}$, the economy converges to the free entry regime with high growth. Thus, here, an outcome that maximizes the utilitarian social welfare is not chosen if $L_{1}>L^{*}$.

In the political equilibrium, the outcome balances the high growth because of entry (the benefit of free entry regime) and the profit from monopoly (the benefit of entry blocking 
regime). From the social perspective, the monopoly is costly, in particular for the workers. Therefore, the social planner's solution favors the free entry regime compared to the political equilibrium outcome. To illustrate the discrepancy of interests, Table 2 compares the steadystate welfare (discounted sum of utility) for workers $(w)$, entrepreneurs and policymakers combined $(e$ and $p)$, and the social welfare (total) starting from $\bar{A}_{0}=1$. The workers are better off in the free entry steady state, while the active participants of the political process (entrepreneurs and policymakers) are better off in the entry blocking steady state.

\section{B Proofs}

Before the proof of Proposition 3, we need one technical lemma.

Lemma B.1 For all integer $i, j, k$ and action $a$ :

$$
i\left(1-\delta_{a}\right) Q_{i-1, j-1}^{k-1}(a)+(k-i) \lambda_{a} Q_{i, j-1}^{k-1}(a)=j Q_{i, j}^{k}(a)
$$

and

$$
i \delta_{a} Q_{i-1, j}^{k-1}(a)+(k-i)\left(1-\lambda_{a}\right) Q_{i, j}^{k-1}(a)=(k-j) Q_{i, j}^{k}(a)
$$

hold.

Proof. Suppose that there are $i$ leaders and $k-i$ followers in the economy. Without loss of generality, index the leaders $h=1, \ldots, i$ and the followers $h=i+1, \ldots, k$. Let $W_{h}$ be a random variable that is 1 if in the next period (i) the firm $h$ is a leader and (ii) there are exactly $j$ leaders in the economy. Define $X \equiv \sum_{h=1}^{i} W_{h}, Y \equiv \sum_{h=i+1}^{k} W_{h}$, and $Z \equiv \sum_{h=1}^{k} W_{h}$. Then the expected values of these, conditional on there being $i$ leaders and $k-i$ followers today, is

$$
\begin{aligned}
E[Z]= & E\left[E\left[\sum_{h=1}^{k} W_{h} \mid \# \text { leaders next period }=j\right]\right] \\
= & E\left[\sum_{h=1}^{k} W_{h} \mid \# \text { leaders next period }=j\right] \times \operatorname{Pr}[\# \text { leaders next period }=j] \\
& +E\left[\sum_{h=1}^{k} W_{h} \mid \# \text { leaders next period } \neq j\right] \times \operatorname{Pr}[\# \text { leaders next period } \neq j] \\
= & j Q_{i, j}^{k}(a),
\end{aligned}
$$




$$
E[X]=E\left[\sum_{h=1}^{i} W_{h}\right]=i E\left[W_{1}\right]=i\left(1-\delta_{a}\right) Q_{i-1, j-1}^{k-1}(a)
$$

and

$$
E[Y]=E\left[\sum_{h=i+1}^{k} W_{h}\right]=(k-i) E\left[W_{i+1}\right]=j \lambda_{a} Q_{i, j-1}^{k-1}(a) .
$$

Then the first statement follows from the fact that $X+Y=Z$, and thus $E[X]+E[Y]=E[Z]$.

The proof of the second statement is analogous.

Proof of Proposition 3. The proof is an adaptation of the proof of Theorem 4 in Bergemann and Välimäki (2003). The proof first aims to establish the existence of a fixed point in the mapping from the list of net payoffs by firms and the policymaker, $\left\{\left\{n_{\ell}\left(L^{i}\right)\right\}_{i=0}^{m}\right.$, $\left.\left\{n_{f}\left(L^{i}\right)\right\}_{i=0}^{m},\left\{G\left(L^{i}\right)\right\}_{i=0}^{m}\right\}$ to itself. That is, given $\left\{\left\{n_{\ell}\left(L^{i}\right)\right\}_{i=0}^{m},\left\{n_{f}\left(L^{i}\right)\right\}_{i=0}^{m},\left\{G\left(L^{i}\right)\right\}_{i=0}^{m}\right\}$ and using these as the continuation payoffs, one can assign (using a particular procedure) the current values (we will call them $\left\{\left\{n_{\ell}^{\prime}\left(L^{i}\right)\right\}_{i=0}^{m},\left\{n_{f}^{\prime}\left(L^{i}\right)\right\}_{i=0}^{m},\left\{G^{\prime}\left(L^{i}\right)\right\}_{i=0}^{m}\right\}$ ) that correspond to a Truthful Markov Perfect Equilibrium to firms and policymakers. If we can find a current list of (net) payoffs that are identical to the list of payoffs we started with (i.e. the list of payoffs has a fixed point), the list constitutes the Truthful Markov Perfect Equilibrium (by construction), and we are done with the existence proof. In establishing the existence of the fixed point, we utilize Brouwer's fixed point theorem.

Define $K=\max \left\{\pi_{\ell}, \pi_{f}\right\} /(1-\beta)$. Let $n_{\ell}=\left\{n_{\ell}\left(L^{i}\right)\right\}_{i=0}^{m}, n_{f}=\left\{n_{f}\left(L^{i}\right)\right\}_{i=0}^{m}, G=$ $\left\{G\left(L^{i}\right)\right\}_{i=0}^{m}$ be arbitrary real $(m+1)$-dimensional vectors such that $n_{\ell} \in[0, K]^{m+1}, n_{f} \in$ $[0, K]^{m+1}$, and $G \in[0, K /(1-\beta)]^{m+1}$.

Define $v_{\ell}\left(a, L^{i}\right)$ and $v_{f}\left(a, L^{i}\right)$ as in the body of the paper and let $V_{\ell}\left(a, L^{i}\right)=L^{i} v_{\ell}\left(a, L^{i}\right)$, $V_{f}\left(a, L^{i}\right)=\left(1-L^{i}\right) v_{f}\left(a, L^{i}\right)$. Also, let

$$
V_{f, \ell}\left(a, L^{i}\right)=V_{\ell}\left(a, L^{i}\right)+V_{f}\left(a, L^{i}\right)
$$

and

$$
V_{\emptyset}\left(a, L^{i}\right)=0
$$


From (14), (15), and Lemma B.1,

$$
V_{f, \ell}\left(a, L^{i}\right)=\left(1-\delta_{a}\right) L^{i} \pi_{\ell}+\lambda_{a}\left(1-L^{i}\right) \pi_{f}+\beta \sum_{j=0}^{m} P_{i, j}(a)\left[L^{j} n_{\ell}\left(L^{j}\right)+\left(1-L^{j}\right) n_{f}\left(L^{j}\right)\right] .
$$

Also let

$$
W_{S}\left(L^{i}\right)=\max _{a \in\{e, b\}} V_{S}\left(a, L^{i}\right)+\beta \sum_{j=0}^{m} P_{i, j}(a) G\left(L^{j}\right),
$$

where $S \subseteq\{f, \ell\}$. By the Maximum Theorem, $W_{S}$ is continuous in the vectors $n_{\ell}, n_{f}$, and $G$.

Set

$$
\begin{gathered}
N_{\ell}^{\prime}\left(L^{i}\right)=W_{\ell, f}\left(L^{i}\right)-W_{f}\left(L^{i}\right), \\
N_{f}^{\prime}\left(L^{i}\right)=\min \left\{W_{\ell, f}\left(L^{i}\right)-W_{\emptyset}\left(L^{i}\right)-N_{\ell}^{\prime}\left(L^{i}\right), W_{\ell, f}\left(L^{i}\right)-W_{\ell}\left(L^{i}\right)\right\},
\end{gathered}
$$

and

$$
G^{\prime}\left(L^{i}\right)=W_{\ell, f}\left(L^{i}\right)-N_{\ell}^{\prime}\left(L^{i}\right)-N_{f}^{\prime}\left(L^{i}\right)
$$

By Theorem 2 in Bernheim and Whinston (1986) (since these net payoffs are in the Pareto efficient frontier in their language), these payoff vectors can be supported by an action $a^{*}\left(L^{i}\right)$ that maximizes $V_{\ell, f}\left(a, L^{i}\right)+\beta \sum_{j=0}^{m} P_{i, j}(a) G\left(L^{j}\right)$ and a pair of bidding schedules that constitute a truthful Nash equilibrium. By the Maximum Theorem, the payoffs are continuous in the continuation vectors (that is, $\left.\left\{\left\{n_{\ell}\left(L^{i}\right)\right\}_{i=0}^{m},\left\{n_{f}\left(L^{i}\right)\right\}_{i=0}^{m},\left\{G\left(L^{i}\right)\right\}_{i=0}^{m}\right\}\right)$. Define $n_{\ell}^{\prime}\left(L^{i}\right)=N_{\ell}^{\prime}\left(L^{i}\right) / L^{i}$ if $L^{i}>0$ and $n_{\ell}^{\prime}(0)=0$; and $n_{f}^{\prime}\left(L^{i}\right)=N_{f}^{\prime}\left(L^{i}\right) /\left(1-L^{i}\right)$ if $L^{i}<1$ and $n_{f}^{\prime}(1)=0$. Then $n_{j}^{\prime}(L)$ is continuous in the continuation vectors. Next, we show that $n_{j}^{\prime} \in[0, K]^{m+1}(j \in\{\ell, f\})$ and $G \in[0, K /(1-\beta)]^{m+1}$.

$n_{\ell}^{\prime}(0)=0$ by construction. When $L^{i}>0, n_{\ell}^{\prime}\left(L^{i}\right)=N_{\ell}^{\prime}\left(L^{i}\right) / L^{i}=\left(V_{\ell}\left(a^{*}, L^{i}\right)-R_{\ell}\left(a^{*}\right)\right) / L^{i} \leq$ $V_{\ell}\left(a^{*}, L^{i}\right) / L^{i}=v_{\ell}\left(a^{*}, L^{i}\right)$. From (14) and the definition of $K$,

$$
\begin{aligned}
v_{\ell}\left(a, L^{i}\right) & =\left(1-\delta_{a}\right) \pi_{\ell}+\beta\left(1-\delta_{a}\right) \sum_{j=1}^{m} Q_{i-1, j-1}^{m-1}(a) n_{\ell}\left(L^{j}\right)+\beta \delta_{a} \sum_{j=0}^{m-1} Q_{i-1, j}^{m-1}(a) n_{f}\left(L^{j}\right) \\
& \leq(1-\beta) K+\beta\left(1-\delta_{a}\right) \sum_{j=1}^{m} Q_{i-1, j-1}^{m-1}(a) K+\beta \delta_{a} \sum_{j=0}^{m-1} Q_{i-1, j}^{m-1}(a) K \\
& =(1-\beta) K+\beta\left(1-\delta_{a}\right) K+\beta \delta_{a} K \\
& =K .
\end{aligned}
$$


Since a bidding schedule $R_{\ell}(e)=0, R_{\ell}(b)=0$ is feasible and $\left\{N_{\ell}^{\prime}\left(L^{i}\right)\right\}$ is supported as a Nash equilibrium, we have that:

$$
n_{\ell}^{\prime}\left(L^{i}\right)=N_{\ell}^{\prime}\left(L^{i}\right) / L^{i} \geq \min _{a} V_{\ell}\left(a, L^{i}\right) / L^{i}=\min _{a} v_{\ell}\left(a, L^{i}\right) \geq 0
$$

Then we have that $n_{\ell}^{\prime}\left(L^{i}\right) \in[0, K]$ for all $L^{i}$. With similar steps, one can also prove that $n_{f}\left(L^{i}\right) \in[0, K]$. Finally, for $G^{\prime}\left(L^{i}\right)$ we know that:

$$
G^{\prime}\left(L^{i}\right)=W_{\ell, f}\left(L^{i}\right)-N_{\ell}^{\prime}\left(L^{i}\right)-N_{f}^{\prime}\left(L^{i}\right) \leq W_{\ell, f}\left(L^{i}\right)
$$

$W_{\ell, f}\left(L^{i}\right)$ satisfies the following inequality:

$$
\begin{aligned}
W_{\ell, f}= & \max _{a \in\{e, b\}}\left\{\left(1-\delta_{a}\right) L^{i} \pi_{\ell}+\lambda_{a}\left(1-L^{i}\right) \pi_{f}+\beta \sum_{j=0}^{m} P_{i, j}(a)\left[L^{i} n_{\ell}\left(L^{i}\right)+\left(1-L^{i}\right) n_{f}\left(L^{i}\right)\right]\right. \\
& \left.\quad+\beta \sum_{j=0}^{m} P_{i, j}(a) G\left(L^{i}\right)\right\} \\
\leq & \max _{a \in\{e, b\}}\left\{(1-\beta) K+\beta \sum_{j=0}^{m} P_{i, j}(a) K+\beta \sum_{j=0}^{m} P_{i, j}(a) K /(1-\beta)\right\} \\
= & K /(1-\beta) .
\end{aligned}
$$

Therefore, $G^{\prime}\left(L^{i}\right) \leq K /(1-\beta)$.

On the other hand, (B.1) and (B.2) imply that $N_{\ell}^{\prime}\left(L^{i}\right)+N_{f}^{\prime}\left(L^{i}\right) \leq W_{\ell, f}\left(L^{i}\right)-W_{\emptyset}\left(L^{i}\right)$. So,

$$
G^{\prime}\left(L^{i}\right)=W_{\ell, f}\left(L^{i}\right)-N_{\ell}^{\prime}\left(L^{i}\right)-N_{f}^{\prime}\left(L^{i}\right) \geq W_{\emptyset}\left(L^{i}\right)=\beta \sum_{j=0}^{m} P_{i, j}(a) G\left(L^{i}\right) \geq 0 .
$$

Therefore, the mapping described above, from $\left\{\left\{n_{\ell}\left(L^{i}\right)\right\}_{i=0}^{m},\left\{n_{f}\left(L^{i}\right)\right\}_{i=0}^{m},\left\{G\left(L^{i}\right)\right\}_{i=0}^{m}\right\}$ to $\left\{\left\{n_{\ell}^{\prime}\left(L^{i}\right)\right\}_{i=0}^{m},\left\{n_{f}^{\prime}\left(L^{i}\right)\right\}_{i=0}^{m},\left\{G^{\prime}\left(L^{i}\right)\right\}_{i=0}^{m}\right\}$, is continuous and maps the compact and convex set $[0, K]^{m+1} \times[0, K]^{m+1} \times[0, K /(1-\beta)]^{m+1}$ to itself. Then, by Brouwer's fixed point theorem, there exists a vector of payoffs that is a fixed point of the mapping described above. By Theorem 2 in Bernheim and Whinston (1986), these are equilibrium payoffs of the Truthful Nash Equilibrium of the one-shot game with specified continuation payoffs. Therefore, they are payoffs of a Truthful Markov Perfect Equilibrium. 
Next, we show the second statement. Let $S^{m *}\left(L^{i}\right) \equiv L^{i} n_{\ell}^{*}\left(L^{i}\right)+\left(1-L^{i}\right) n_{f}^{*}\left(L^{i}\right)+G^{*}\left(L^{i}\right)$. (The* denotes that these functions constitute a Truthful Markov Perfect Equilibrium.) Then,

$$
\begin{aligned}
S^{m *}\left(L^{i}\right)= & L^{i} n_{\ell}^{*}\left(L^{i}\right)+\left(1-L^{i}\right) n_{f}^{*}\left(L^{i}\right)+G^{*}\left(L^{i}\right) \\
= & V_{\ell}^{*}\left(a^{m *}\left(L^{i}\right), L^{i}\right)-R_{\ell}^{*}\left(a^{m *}\left(L^{i}\right), L^{i}\right)+V_{f}^{*}\left(a^{m *}\left(L^{i}\right), L^{i}\right)-R_{f}^{*}\left(a^{m *}\left(L^{i}\right), L^{i}\right) \\
& +\sum_{s \in\{\ell, f\}} R_{s}^{*}\left(a^{m *}\left(L^{i}\right), L^{i}\right)+\beta \sum_{i, j}\left(a^{m *}\left(L^{i}\right)\right) G^{*}\left(L^{j}\right) \\
= & V_{\ell}^{*}\left(a^{m *}\left(L^{i}\right), L^{i}\right)+V_{f}^{*}\left(a^{m *}\left(L^{i}\right), L^{i}\right)+\beta \sum_{j=0}^{m} P_{i, j}\left(a^{m *}\left(L^{i}\right)\right) G^{*}\left(L^{j}\right) \\
= & \left(1-\delta_{a^{m *}\left(L^{i}\right)}\right) L^{i} \pi_{\ell}+\lambda_{a^{m *}\left(L^{i}\right)}\left(1-L^{i}\right) \pi_{f} \\
& +\beta \sum_{j=0}^{m} P_{i, j}\left(a^{*}\left(L^{i}\right)\right)\left[L^{j} n_{\ell}^{*}\left(L^{j}\right)+\left(1-L^{j}\right) n_{f}^{*}\left(L^{j}\right)+G^{*}\left(L^{j}\right)\right] \\
= & \max _{a \in\{e, b\}}\left\{\left(1-\delta_{a}\right) L^{i} \pi_{\ell}+\lambda_{a}\left(1-L^{i}\right) \pi_{f}\right. \\
& \left.\quad+\beta \sum_{j=0}^{m} P_{i, j}(a)\left[L^{j} n_{\ell}^{*}\left(L^{j}\right)+\left(1-L^{j}\right) n_{f}^{*}\left(L^{j}\right)+G^{*}\left(L^{j}\right)\right]\right\} \\
= & \max _{a \in\{e, b\}}\left\{\left(1-\delta_{a}\right) L^{i} \pi_{\ell}+\lambda_{a}\left(1-L^{i}\right) \pi_{f}+\beta \sum_{j=0}^{m} P_{i, j}(a) S^{m *}\left(L^{j}\right)\right\},
\end{aligned}
$$

where the fifth equation is from Theorem 2 in Bernheim and Whinston (1986) (there, it is shown that the equilibrium action $a^{m *}\left(L^{i}\right)$ attains $\left.W_{\ell, f}\left(L^{i}\right)\right)$.

Proof of Proposition 4. Define $Z(L) \equiv L n_{\ell}(L)+(1-L) n_{f}(L)+G(L)$. In equilibrium, the following holds. (Superscript ${ }^{*}$ is added to clarify that all value functions are in equilibrium.)

$$
\begin{aligned}
Z^{*}(L) & =L n_{\ell}^{*}(L)+(1-L) n_{f}^{*}(L)+G^{*}(L) \\
& =V_{\ell}^{*}\left(a^{*}(L), L\right)+V_{f}^{*}\left(a^{*}(L), L\right)+\beta G^{*}\left(\mathcal{L}\left(a^{*}(L), L\right)\right) \\
& =\left(1-\delta_{a^{*}(L)}\right) L \pi_{\ell}+\lambda_{a^{*}(L)}(1-L) \pi_{f}+\beta Z^{*}\left(\mathcal{L}\left(a^{*}(L), L\right)\right),
\end{aligned}
$$

where the second equality uses the fact that the payment that the coalitions pay is equal to the payment that the policymaker receives in equilibrium, and the third equality uses the definitions of $v_{\ell}(a, L), v_{f}(a, L), \mathcal{L}(a, L)$, and $Z(L)$.

Now, notice that in the Truthful Markov Perfect Equilibrium (Proposition 2), $V_{\ell}^{*}\left(a^{*}(L), L\right)+$ $V_{f}^{*}\left(a^{*}(L), L\right)+\beta G^{*}\left(\mathcal{L}\left(a^{*}(L), L\right)\right) \geq V_{\ell}^{*}(a, L)+V_{f}^{*}(a, L)+\beta G^{*}(\mathcal{L}(a, L))$ for $a \neq a^{*}(L)$. From 
the above equation, this means that $\left(1-\delta_{a^{*}(L)}\right) L \pi_{\ell}+\lambda_{a^{*}(L)}(1-L) \pi_{f}+\beta Z^{*}\left(\mathcal{L}\left(a^{*}(L), L\right)\right) \geq$ $\left(1-\delta_{a}\right) L \pi_{\ell}+\lambda_{a}(1-L) \pi_{f}+\beta Z^{*}(\mathcal{L}(a, L))$ for $a \neq a^{*}(L)$. This implies that $Z^{*}(L)$ satisfies

$$
Z^{*}(L)=\max _{a}\left\{\left(1-\delta_{a}\right) L \pi_{\ell}+\lambda_{a}(1-L) \pi_{f}+\beta Z^{*}(\mathcal{L}(a, L))\right\}
$$

which means that $Z^{*}(L)$ is a solution to the functional equation $(17)$ and $a^{*}(L)$ is its policy function.

Proof of Proposition 5. The operator satisfies monotonicity and discounting, therefore by Blackwell's theorem it is a contraction on the space of bounded functions (see, for example, Stokey, Lucas, and Prescott (1989)). Let $f$ be a convex function. Then $p_{a} L+q_{a}+\beta f(\mathcal{L}(a, L))$ is convex in $L$ as composition of a convex and an affine function. Therefore $T f$ is convex (as a maximum of convex functions) if $f$ is convex. Then since the space of bounded convex functions is closed, the unique fixed point is bounded and convex, and therefore continuous.

The statement that $S$ is differentiable everywhere except on an (at most) countable set is equivalent to Theorem 25.3 in Rockafellar (1970).

Suppose that $p_{b} /\left(1-\beta \gamma_{b}\right) \geq p_{e} /\left(1-\beta \gamma_{e}\right)$. Let $A$ be the set of bounded convex functions $f$ such that $f^{\prime}(L) \in\left[p_{e} /\left(1-\beta \gamma_{e}\right), p_{b} /\left(1-\beta \gamma_{b}\right)\right]$. If $f \in A, T f$ is differentiable almost everywhere, and where it is differentiable

$$
(T f)^{\prime}(L)=p_{a}+\beta \gamma_{a} f^{\prime}(\mathcal{L}(a, L))
$$

holds, where $a$ is the optimal action at $L$. The derivative can be bounded as

$$
\begin{aligned}
(T f)^{\prime}(L) & =p_{a}+\beta \gamma_{a} f^{\prime}(\mathcal{L}(a, L)) \\
& \leq p_{a}+\beta \gamma_{a} \frac{p_{b}}{1-\beta \gamma_{b}} \\
& =\frac{p_{b}}{1-\beta \gamma_{b}}+\left(1-\beta \gamma_{a}\right)\left(\frac{p_{a}}{1-\beta \gamma_{a}}-\frac{p_{b}}{1-\beta \gamma_{b}}\right) \\
& \leq \frac{p_{b}}{1-\beta \gamma_{b}} .
\end{aligned}
$$

In a similar fashion, we establish that $(T f)^{\prime}(L) \geq p_{e} /\left(1-\beta \gamma_{e}\right)$. So the operator $T$ maps $A$ to itself. We will show that $A$ is closed in the sup norm.

Let $f_{i}$ be a sequence of functions, $f_{i} \in A$, that converges to $f$ in the sup norm. Since the functions are convex and continuous, the condition $f \in A$ is equivalent to $\left(f\left(L_{2}\right)-\right.$ 
$\left.f\left(L_{1}\right)\right) /\left(L_{2}-L_{1}\right) \in\left[p_{e} /\left(1-\beta \gamma_{e}\right), p_{b} /\left(1-\beta \gamma_{b}\right)\right]$ for $L_{1} \neq L_{2}$. Since $\left(f_{i}\left(L_{2}\right)-f_{i}\left(L_{1}\right)\right) /\left(L_{2}-L_{1}\right) \in$ $\left[p_{e} /\left(1-\beta \gamma_{e}\right), p_{b} /\left(1-\beta \gamma_{b}\right)\right]$ for all $f_{i}$, and $\left(f\left(L_{2}\right)-f\left(L_{1}\right)\right) /\left(L_{2}-L_{1}\right)$ is continuous in $f\left(L_{1}\right)$ and $f\left(L_{2}\right), f(L)$ also satisfies this condition. Therefore $f \in A$, and thus $A$ is closed in the sup norm. Then by the Contraction Mapping Theorem, $S \in A$.

The case when $p_{b} /\left(1-\beta \gamma_{b}\right)<p_{e} /\left(1-\beta \gamma_{e}\right)$ is analogous.

Proof of Statement 1 of Proposition 6. Let $T$ be the mapping defined by the righthand side of (17). Since $T$ is a contraction, if we show that $T S_{e}=S_{e}$, we prove that $S_{e}=S$ and that $e \in a^{*}(L)$. From the definitions we see that $T S_{e}(L)=\max \left\{S_{e}(L), S_{b, e}(L)\right\}$. Then it will be sufficient to show that $g(L) \equiv S_{e}(L)-S_{b, e}(L)>0$ for all $L$. We will show this by proving (i) $g(0)>0$ and (ii) $g^{\prime}(L) \geq 0$. First, (i) is shown as

$$
\begin{aligned}
g(0) & =\lambda_{e} \pi_{f}-\lambda_{b} \pi_{f}+\beta\left(S_{e}\left(\lambda_{e}\right)-S_{e}\left(\lambda_{b}\right)\right) \\
& =\left(\lambda_{e}-\lambda_{b}\right)\left(\pi_{f}+\beta \frac{p_{e}}{1-\beta \gamma_{e}}\right) \\
& =\left(\lambda_{e}-\lambda_{b}\right) \frac{\beta\left(1-\delta_{e}\right) \pi_{\ell}+\left(1-\beta+\beta \delta_{e}\right) \pi_{f}}{1-\beta \gamma_{e}}>0,
\end{aligned}
$$

where we used the fact that $S_{e}$ is linear and the definitions. Second, (ii) is shown as

$$
g^{\prime}(L)=\frac{p_{e}}{1-\beta \gamma_{e}}-p_{b}-\beta \gamma_{b} \frac{p_{e}}{1-\beta \gamma_{e}}=\left(1-\beta \gamma_{b}\right)\left(\frac{p_{e}}{1-\beta \gamma_{e}}-\frac{p_{b}}{1-\beta \gamma_{b}}\right) \geq 0
$$

\section{Proof of Statement 2 of Proposition 6.}

(a) Direct calculation yields:

$$
S_{b}^{\prime}(L)-S_{e, b}^{\prime}(L)=\left(1-\beta \gamma_{e}\right)\left(\frac{p_{b}}{1-\beta \gamma_{b}}-\frac{p_{e}}{1-\beta \gamma_{e}}\right)>0
$$

We will divide this case into two subcases: (i) $S_{b}(0) \geq S_{e, b}(0)$ and (ii) $S_{b}(0)<S_{e, b}(0)$.

First, suppose that $S_{b}(0) \geq S_{e, b}(0)$. Then from the inequality above, it follows that $S_{b}(L) \geq S_{e, b}(L)$ for all $L$. Therefore $T S_{b}(L)=\max \left\{S_{b}(L), S_{e, b}(L)\right\}=S_{b}(L)$, so $S(L)=$ $S_{b}(L)$ and $b \in a^{*}(L)$ for all $L \in[0,1]$. We can consider this as a case where $L^{*}<0$. 
Second, suppose that $S_{b}(0)<S_{e, b}(0)$. Then (since both $S_{b}(0)$ and $S_{e, b}$ are linear) there exists some $L^{*} \in\left(0, \bar{L}_{e}\right]$ such that $S_{b}\left(L^{*}\right)=S_{e, b}\left(L^{*}\right)$. We will show that the threshold policy " $a(L)=e$ if $L<L^{*}$ and $a(L)=b$ if $L \geq L^{*}$ " is optimal. Let $\hat{S}(L)$ be the value of following this policy.

Let $\mathcal{L}^{(i)}(a, L)$ be the value of $L_{t+i}$ when the value of $L_{t}$ is $L$ and the policy $a$ is conducted at time $t, t+1, \ldots, t+i-1$. Thus $\mathcal{L}^{(1)}(a, L)=\mathcal{L}(a, L), \mathcal{L}^{(2)}(a, L)=\mathcal{L}(a, \mathcal{L}(a, L))$, and so on.

Let $n=\min \left\{i: \mathcal{L}^{(i)}(e, 0) \geq L^{*}\right\}$. That is, $n$ is the minimum number of steps it takes to reach a value of $L$ larger than $L^{*}$ when $L$ starts from zero and the policy $e$ is taken. Then define $L^{(i)}, i=1, \ldots, n-1$ by $\mathcal{L}^{(i)}\left(e, L^{(i)}\right)=L^{*}$. That is, $L^{(i)}$ is the exact starting value of $L$ which, after $i$ periods of policy $e$, can lead to $L^{*}$. (Also let $L^{(0)}=L^{*}$ and $L^{(n)}=0$ when necessary.) $L^{(i)}$ partitions the interval $[0,1]$ into $\left[0, L^{(n-1)}\right),\left[L^{(n-1)}, L^{(n-2)}\right),\left[L^{(n-2)}, L^{(n-3)}\right), \ldots,\left[L^{(1)}, L^{*}\right),\left[L^{*}, 1\right]$.

First consider $L$ in the interval $\left[L^{*}, 1\right]$. Since $\bar{L}_{b} \geq L^{*}, \hat{S}(L)=S_{b}(L)$. Second, consider $L \in\left[L_{1}, L^{*}\right)$. Since $L$ becomes larger than $L^{*}$ after one period of policy e, $\hat{S}(L)=$ $S_{e, b}(L)$. By definition $S_{b}\left(L^{*}\right)=S_{e, b}\left(L^{*}\right)$, so $\hat{S}$ is continuous at $L^{*}$. Since $\hat{S}(L)=p_{e} L+$ $q_{e}+\beta \hat{S}(\mathcal{L}(e, L))$ for $L<L^{*}$, by induction, it follows that $\hat{S}$ is continuous everywhere. $\hat{S}(L)$ is differentiable everywhere, except at $L^{(i)}$ and $L^{*}$. Next consider the interval $L \in\left(L^{(i)}, L^{(i-1)}\right)$,

$$
\hat{S}^{\prime}(L)=\sum_{j=1}^{i} p_{e}\left(\beta \gamma_{e}\right)^{j-1}+\left(\beta \gamma_{e}\right)^{i} \frac{p_{b}}{1-\beta \gamma_{b}}=\left(1-\left(\beta \gamma_{e}\right)^{i}\right) \frac{p_{e}}{1-\beta \gamma_{e}}+(\beta \gamma)^{i} \frac{p_{b}}{1-\beta \gamma_{b}}
$$

Then $\hat{S}^{\prime}(L)$ is increasing and $\hat{S}^{\prime}(L) \leq p_{b} /\left(1-\beta \gamma_{b}\right)$.

Let $T$ be the mapping defined by the right-hand side of (17). Now we show that $T \hat{S}=\hat{S}$. Define

$$
g(L) \equiv p_{e} L+q_{e}+\beta \hat{S}(\mathcal{L}(e, L))-p_{b} L-q_{b}-\beta \hat{S}(\mathcal{L}(b, L))
$$


Suppose that $L \geq L^{*}$. Then $\mathcal{L}(a, L) \geq L^{*}$. Therefore:

$$
\begin{aligned}
T \hat{S}(L) & =\max \left\{p_{e} L+q_{e}+\beta \hat{S}(\mathcal{L}(e, L)), p_{b} L+q_{b}+\beta \hat{S}(\mathcal{L}(b, L))\right\} \\
& =\max \left\{S_{e, b}(L), S_{b}(L)\right\} \\
& =S_{b}(L) \\
& =\hat{S}(L)
\end{aligned}
$$

Note that here $g(L)=S_{e, b}(L)-S_{b}(L)$ is decreasing in $L$ from (B.3). Suppose that $L<L^{*}$. Then $\hat{S}(L)=p_{e} L+q_{e}+\beta \hat{S}(\mathcal{L}(e, L))$. We will show that $g(L)$ defined in (B.4) is positive. Here, $g(L)=\hat{S}(L)-p_{b} L-q_{b}-\beta \hat{S}(\mathcal{L}(b, L))$. By the choice of $L^{*}, g\left(L^{*}\right)=0$. Since $g$ is continuous and differentiable always everywhere, it is sufficient to show that $g^{\prime}(L)<0$ where it is defined.

$$
\begin{aligned}
g^{\prime}(L) & =\hat{S}^{\prime}(L)-p_{b}-\beta \gamma_{b} \hat{S}^{\prime}(\mathcal{L}(b, L)) \\
& \leq \hat{S}^{\prime}(L)-p_{b}-\beta \gamma_{b} \hat{S}^{\prime}(L) \\
& =\left(1-\beta \gamma_{b}\right)\left(\hat{S}^{\prime}(L)-\frac{p_{b}}{1-\beta \gamma_{b}}\right)<0,
\end{aligned}
$$

where we used the fact that $\mathcal{L}(b, L)>L$ and $\hat{S}^{\prime}(L)$ is increasing.

(b) The proof in this case is symmetric to case (a). Note that $g(L)$ defined as (B.4) is decreasing here as well.

(c) $S_{b}\left(\bar{L}_{e}\right)<S_{e, b}\left(\bar{L}_{e}\right)=p_{e} \bar{L}_{e}+q_{e}+\beta S_{b}\left(\bar{L}_{e}\right)$ implies that $S_{b}\left(\bar{L}_{e}\right)<\left(p_{e} \bar{L}_{e}+q_{e}\right) /(1-\beta)=$ $S_{e}\left(\bar{L}_{e}\right)$. Similarly, $S_{b}\left(\bar{L}_{b}\right)>S_{e}\left(\bar{L}_{b}\right)$. Since $S_{b}$ and $S_{e}$ are both linear, there exists $L^{*}$ defined by

$$
S_{b}\left(L^{*}\right)=S_{e}\left(L^{*}\right)
$$

We will show that policy $a(L)=e$ if $L \leq L^{*}, a(L)=b$ if $L \geq L^{*}$ is optimal. The value of following this policy is:

$$
\hat{S}(L)= \begin{cases}S_{e}(L) & \text { if } L<L^{*} \\ S_{b}(L) & \text { if } L \geq L^{*}\end{cases}
$$

It is sufficient to show that $T \hat{S}=\hat{S}$. Define $g(L)$ by (B.4). It will be sufficient to show that $g(L) \geq 0$ on $\left[0, L^{*}\right]$ and $g(L) \leq 0$ on $\left[L^{*}, 1\right]$. By the choice of $L^{*}, g\left(L^{*}\right)=0$. It is straightforward to show that $\hat{S}(L)$ is continuous, therefore $g(L)$ is also continuous. 
Since $g(L)$ is continuous, it will be sufficient to show that $g^{\prime}(L)<0$ where it is defined; $g^{\prime}(L)$ can be calculated as

$$
g^{\prime}(L)=p_{e}+\beta \gamma_{e} \hat{S}^{\prime}(\mathcal{L}(e, L))-p_{b}-\beta \gamma_{b} \hat{S}^{\prime}(\mathcal{L}(b, L))
$$

If $L \leq L^{*}, \mathcal{L}(e, L)<L^{*}$, so

$$
g^{\prime}(L) \leq p_{e}+\beta \gamma_{e} \frac{p_{e}}{1-\beta \gamma_{e}}-p_{b}-\beta \gamma_{b} \frac{p_{e}}{1-\beta \gamma_{e}}=\left(1-\beta \gamma_{b}\right)\left(\frac{p_{e}}{1-\beta \gamma_{e}}-\frac{p_{b}}{1-\beta \gamma_{b}}\right)<0 .
$$

If $L>L^{*}, \mathcal{L}(b, L)>L^{*}$, so

$$
g^{\prime}(L) \leq p_{e}+\beta \gamma_{e} \frac{p_{b}}{1-\beta \gamma_{b}}-p_{b}-\beta \gamma_{b} \frac{p_{b}}{1-\beta \gamma_{b}}=\left(1-\beta \gamma_{e}\right)\left(\frac{p_{e}}{1-\beta \gamma_{e}}-\frac{p_{b}}{1-\beta \gamma_{b}}\right)<0
$$

\section{Proof of Statement 3 of Proposition 6.}

(a) Identical to the proof of case (a) of statement 2 of Proposition 6. Note that $g(L)$ defined as (B.4) is decreasing here as well.

(b) Identical to the proof of case (b) of statement 2 of Proposition 6. Note that $g(L)$ defined as (B.4) is decreasing here as well.

(c) Again, let $T$ be the mapping defined by the right-hand side of (17). Now define $g(L)$ by

$$
g(L) \equiv p_{e} L+q_{e}+\beta S(\mathcal{L}(e, L))-p_{b} L-q_{b}-\beta S(\mathcal{L}(b, L))
$$

Note that the fixed point $S$ is used in the definition. By Proposition $5, g$ is continuous and differentiable everywhere except on a countable set. We will show that there exists a $L^{*} \in\left(\bar{L}_{b}, \bar{L}_{e}\right)$ where $g\left(L^{*}\right)=0, g(L)>0$ for $L \in\left[0, L^{*}\right)$, and $g(L)<0$ for $L \in\left(L^{*}, 1\right]$, which implies the proposition. 
The bounds on $S^{\prime}(L)$ (from Proposition 5) imply that

$$
\begin{aligned}
g^{\prime}(L) & =p_{e}+\beta \gamma_{e} S^{\prime}(\mathcal{L}(e, L))-p_{b} L-\beta \gamma_{b} S^{\prime}(\mathcal{L}(b, L)) \\
& \leq p_{e}+\beta \gamma_{e} \frac{p_{b}}{1-\beta \gamma_{b}}-p_{b}-\beta \gamma_{b} \frac{p_{e}}{1-\beta \gamma_{e}} \\
& =\left(1-\beta \gamma_{e}-\beta \gamma_{b}\right)\left(\frac{p_{e}}{1-\beta \gamma_{e}}-\frac{p_{b}}{1-\beta \gamma_{b}}\right) \\
& <0 .
\end{aligned}
$$

Define the set $B$ as $B \equiv\left\{L \in\left(\bar{L}_{b}, \bar{L}_{e}\right): g(L)=0\right\}$. Suppose $g(L)>0$ for all $L \in$ $\left(\bar{L}_{b}, \bar{L}_{e}\right)$. Then for all $L \in\left(\bar{L}_{b}, \bar{L}_{e}\right), S(L)=p_{e} L+q_{e}+\beta S(\mathcal{L}(e, L))$. Since $\mathcal{L}(e, L) \in$ $\left(\bar{L}_{b}, \bar{L}_{e}\right)$, it then follows that $S(L)=S_{e}(L)$ on $\left(\bar{L}_{b}, \bar{L}_{e}\right)$. Then by continuity, $S\left(\bar{L}_{e}\right)=$ $S_{e}\left(\bar{L}_{e}\right)$. This leads to a contradiction, since by assumption $S_{e}\left(\bar{L}_{e}\right)<S_{b, e}\left(\bar{L}_{e}\right)$.

In a similar fashion, we show that $g(L)<0$ for all $L \in\left(\bar{L}_{b}, \bar{L}_{e}\right)$ is impossible. Therefore $B \neq \emptyset$. Take an arbitrary $L^{*} \in B$. Since $g^{\prime}(L)<0$ almost everywhere, $g(L)>0$ for $L \in\left[0, L^{*}\right)$ and $g(L)<0$ for $L \in\left(L^{*}, 1\right]$. This also shows that $B$ is a singleton.

Before proving Proposition 7, we need some preparations.

Lemma B.2 Let $\eta>0, \epsilon>0$ be arbitrary. For given $L$ and a, there exists $m^{*}$ such that for all $m \geq m^{*}$,

$$
\operatorname{Pr}\left[\left|L_{a}^{\prime}-\mathcal{L}(a, L)\right|<\eta\right]>1-\epsilon, \quad \forall L \in\{0,1 / m, \ldots 1\}, a \in\{e, b\}
$$

where $L_{a}^{\prime}$ is the mass of leaders in the next period if policy a is implemented.

Proof. This is an application of the Weak Law of Large Numbers. Let $X_{a}^{j}$ be a random variable distributed according to a binomial distribution with parameter $\left(1-\delta_{a}\right)$ and $j$ attempts. Let $Y_{a}^{j}$ be a similar random variable with parameter $\lambda_{a}$. Then if the number of leaders is currently $i, L_{a}^{\prime}=\left(X_{a}^{i}+Y_{a}^{m-i}\right) / m$. From the definitions, we see that $\operatorname{var}\left(X_{a}^{i}\right)=$ $i \delta_{a}\left(1-\delta_{a}\right)$ and $\operatorname{var}\left(Y_{a}^{m-i}\right)=(m-i) \lambda_{a}\left(1-\lambda_{a}\right)$. Since the transitions of firms' states are 
independent events,

$$
\operatorname{var}\left(L_{a}^{\prime}\right)=\frac{\operatorname{var}\left(X_{a}^{i}\right)+\operatorname{var}\left(Y_{a}^{m-i}\right)}{m^{2}} \leq \frac{\max \left\{\delta_{a}\left(1-\delta_{a}\right), \lambda_{a}\left(1-\lambda_{a}\right)\right\}}{m} .
$$

The Chebyshev inequality implies that

$$
\operatorname{Pr}\left[\left|L_{a}^{\prime}-\mathcal{L}(a, L)\right|<\eta\right] \geq 1-\frac{\operatorname{var}\left(L_{a}^{\prime}\right)}{\eta^{2}} .
$$

Let $m_{a}^{*}=\left\lceil\max \left\{\delta_{a}\left(1-\delta_{a}\right), \lambda_{a}\left(1-\lambda_{a}\right)\right\} / \epsilon \eta^{2}+1\right\rceil$. Then if $m \geq m_{a}^{*}, \operatorname{var}\left(L_{a}^{\prime}\right) / \eta^{2}<\epsilon$ for any $L$. Then if $m \geq m_{a}^{*}$,

$$
\operatorname{Pr}\left[\left|L_{a}^{\prime}-\mathcal{L}(a, L)\right|<\eta\right]>1-\epsilon, \quad \forall L \in\{0,1 / m, \ldots 1\}
$$

Finally, let $m^{*}=\max \left\{m_{e}^{*}, m_{b}^{*}\right\}$. This $m^{*}$ satisfies all the requirements in the lemma.

Proof of Proposition 7. Let $T^{m}$ and $T$ be the Bellman operators in the right-hand sides of (16) and (17) respectively. For a function $f:[0,1] \rightarrow \Re$, define the function $H^{m} f$ : $\{0,1 / m, \ldots 1\} \rightarrow \Re$ by $H^{m} f(i / m)=f(i / m)$. Defining the sup norm in the standard manner, the proposition is equivalent to $\lim _{m \rightarrow \infty}\left\|H^{m} S-S^{m}\right\|=0$. Since $T^{m}$ is a contraction mapping, $\left\|T^{m} H^{m} S-S^{m}\right\| \leq \beta\left\|H^{m} S-S^{m}\right\|$, where we used $T^{m} S^{m}=S^{m}$. From the triangle inequality, $\left\|T^{m} H^{m} S-H^{m} S\right\|+\left\|T^{m} H^{m} S-S^{m}\right\| \geq\left\|H^{m} S-S^{m}\right\|$. Combining these two, $\left\|H^{m} S-S^{m}\right\| \leq\left\|T^{m} H^{m} S-H^{m} S\right\| /(1-\beta)$ holds. Therefore, it is sufficient to show that $\left\|T^{m} H^{m} S-H^{m} S\right\| \rightarrow 0$ as $m \rightarrow \infty$.

Set some arbitrary $\epsilon^{\prime}>0$, such that $\epsilon^{\prime}<K /(1-\beta)$. Since $S$ is uniformly continuous, there exists some $\eta>0$ such that $\left|L^{\prime}-L^{\prime \prime}\right|<\eta$ implies $\left|S\left(L^{\prime}\right)-S\left(L^{\prime \prime}\right)\right|<\epsilon^{\prime}$ for any $L^{\prime}$ and $L^{\prime \prime}$. Set $\epsilon=(1-\beta) \epsilon^{\prime} /\left(K \beta /(1-\beta)-\beta \epsilon^{\prime}\right)$. Let $m^{*}$ be such that it satisfies the conclusion of Lemma B.2 for these $\eta$ and $\epsilon$. (Note that $m^{*}$ only depends on $\epsilon^{\prime}$ and not on $L^{i}$.) We will show that if $m \geq m^{*}$, then $\left|\left(T^{m} H^{m} S\right)\left(L^{i}\right)-S\left(L^{i}\right)\right|<\epsilon^{\prime}$ for all $L^{i} \in\{0,1 / m, \ldots 1\}$, thus proving the proposition.

Let $f\left(a, L^{i} ; S\right) \equiv\left(1-\delta_{a}\right) L^{i} \pi_{\ell}+\lambda_{a}\left(1-L^{i}\right) \pi_{f}+\beta S\left(\mathcal{L}\left(a, L^{i}\right)\right)$ and $f^{m}\left(a, L^{i} ; S\right) \equiv(1-$ $\left.\delta_{a}\right) L^{i} \pi_{\ell}+\lambda_{a}\left(1-L^{i}\right) \pi_{f}+\beta \sum_{j=0}^{m} p_{i, j}(a) S\left(L^{j}\right)$. Then $\left(T^{m} H^{m} S\right)\left(L^{i}\right)=\max _{a^{\prime}} f^{m}\left(a^{\prime}, L^{i} ; S\right)$ and $S\left(L^{i}\right)=\max _{a^{\prime \prime}} f\left(a^{\prime \prime}, L^{i} ; S\right)$. Thus, if we establish that when $m \geq m^{*}, \mid f^{m}\left(a, L^{i} ; S\right)-$ 
$f\left(a, L^{i} ; S\right) \mid<\epsilon^{\prime}$ holds for all $L^{i}$ and $a$, we are done. (If $f^{m}\left(a, L^{i} ; S\right)$ is within $\epsilon^{\prime}$ band of $f\left(a, L^{i} ; S\right)$, then $\max _{a^{\prime}} f^{m}\left(a^{\prime}, L^{i} ; S\right)$ is within $\epsilon^{\prime}$ band of $\left.\max _{a^{\prime \prime}} f\left(a^{\prime \prime}, L^{i} ; S\right).\right)$

Now, suppose that $m \geq m^{*}$. Pick some $L^{i}$ and $a$. Let $j_{1}=\min \left\{j: j / m \geq \mathcal{L}\left(a, L_{i}\right)-\eta\right\}$ and $j_{2}=\max \left\{j: j / m \leq \mathcal{L}\left(a, L_{i}\right)+\eta\right\}$. By the choice of $m^{*}$, it follows that $\sum_{j=j_{1}}^{j_{2}} p_{i, j}(a) \geq$ $1-\epsilon$ for $m \geq m^{*}$. By the choice of $\eta,\left|S\left(L_{j}\right)-S\left(\mathcal{L}\left(a, L_{i}\right)\right)\right|<\epsilon^{\prime}$ if $j \in\left\{j_{1} \ldots j_{2}\right\}$. Since $S(L) \in[0, K /(1-\beta)],\left|S\left(L^{\prime}\right)-S\left(L^{\prime \prime}\right)\right| \leq K /(1-\beta)$ for any $L^{\prime}$ and $L^{\prime \prime}$. Therefore, for $m \geq m^{*}$,

$$
\begin{aligned}
\mid f\left(a, L_{i}\right. & ; S)-f^{m}\left(a, L_{i} ; S\right) \mid \\
& =\beta\left|\sum_{j \neq\left[j_{1}, j_{2}\right]} p_{i, j}(a)\left(S\left(L_{j}\right)-S\left(\mathcal{L}\left(a, L_{i}\right)\right)\right)+\sum_{j=j_{1}}^{j_{2}} p_{i, j}(a)\left(S\left(L_{j}\right)-S\left(\mathcal{L}\left(a, L_{i}\right)\right)\right)\right| \\
& \leq \beta \sum_{j \neq\left[j_{1}, j_{2}\right]} p_{i, j}(a) \frac{K}{1-\beta}+\beta \sum_{j=j_{1}}^{j_{2}} p_{i, j}(a) \epsilon^{\prime} \\
& \leq \beta \epsilon \frac{K}{1-\beta}+\beta(1-\epsilon) \epsilon^{\prime} \\
& =\epsilon^{\prime}
\end{aligned}
$$

holds. Since $\epsilon^{\prime}$ was arbitrary, we are done.

Proof of Proposition 8. For the first statement of Proposition 6, it is straightforward to show the result using the fact that $S^{m}(L)$ converges to $S(L)$ from Proposition 7 .

Consider the cases of statements 2 and 3 of Proposition 6. Set arbitrary $\mathbf{L}_{l}$ and $\mathbf{L}_{u}$ such that $\mathbf{L}_{l}<L^{*}<\mathbf{L}_{u}$. It will be sufficient to show that for all $m$ sufficiently large, $a^{m *}(L)=e$ if $L \leq \mathbf{L}_{l}$ and $a^{m *}(L)=b$ if $L \geq \mathbf{L}_{u}$.

Define $g(L) \equiv p_{e} L+q_{e}+\beta S(\mathcal{L}(e, L))-p_{b} L-q_{b}-\beta S(\mathcal{L}(b, L))$. This is the same $g(L)$ in the proof of Proposition 6 (i.e. (B.4) and (B.5)), because there we showed that $\hat{S}(L)$ is indeed $S(L)$ for (B.4). In the proof of Proposition 6, we have shown that it is a strictly decreasing function. Analogously, define $g^{m}\left(L^{i}\right) \equiv p_{e} L^{i}+q_{e}-p_{b} L^{i}-q_{b}+\beta \sum_{j=0}^{m}\left[p_{i, j}(e)-p_{i, j}(b)\right] S^{m}\left(L^{j}\right)$. 
Then $a^{m *}(L)=e$ if $g^{m}(L)>0$ and $a^{m *}(L)=b$ if $g^{m}(L)<0$.

$$
\begin{aligned}
\left|g\left(L^{i}\right)-g^{m}\left(L^{i}\right)\right| & =\beta\left|\sum_{j=0}^{m} p_{i, j}(e)\left[S(\mathcal{L}(e, L))-S^{m}\left(L^{j}\right)\right]-\sum_{j=0}^{m} p_{i, j}(b)\left[S(\mathcal{L}(b, L))-S^{m}\left(L^{j}\right)\right]\right| \\
& \leq \sum_{a \in\{e, b\}} \max _{i \in\{0, \ldots, m\}}\left|\sum_{j=0}^{m} p_{i, j}(a)\left[S(\mathcal{L}(a, L))-S^{m}\left(L^{j}\right)\right]\right| \\
& \leq \sum_{a \in\{e, b\}} \max _{i \in\{0, \ldots, m\}}\left|\sum_{j=0}^{m} p_{i, j}(a)\left[S(\mathcal{L}(a, L))-S\left(L^{j}\right)\right]\right|+2\left\|S^{m}-H^{m} S\right\|
\end{aligned}
$$

In the proof of Proposition 7, we showed that both terms converge to 0 as $m \rightarrow \infty$. Therefore, there exists some $M$, such that for all $m \geq M,\left|g(L)-g^{m}(L)\right|<\min \left\{\left|g\left(\mathbf{L}_{l}\right)\right|,\left|g\left(\mathbf{L}_{u}\right)\right|\right\}$.

Then if $L \leq \mathbf{L}_{l}$,

$$
g_{m}(L)=g(L)+g_{m}(L)-g(L) \geq g(L)-\left|g_{m}(L)-g(L)\right|>g(L)-\left|g\left(\mathbf{L}_{l}\right)\right| \geq 0
$$

The last inequality follows since $L \leq \mathbf{L}_{l}<L^{*}, g\left(L^{*}\right)=0$, and $g(L)$ is strictly decreasing. Therefore, $a_{m}^{*}(L)=e$ if $L \leq \mathbf{L}_{l}$. The proof for $L \geq \mathbf{L}_{u}$ is analogous.

Proof of Proposition 9. Choose some natural number $T$ such that $\left(L_{t}-\bar{L}_{e}\right) \gamma_{e}^{T}+\bar{L}_{e}<L^{*}$. Such a $T$ exists since $\gamma_{e}<1$ and $\bar{L}_{e}<L^{*}$.

Let $\tilde{a}_{t+s}, s=0, \ldots, T-1$ be an arbitrary sequence of policies, and $\tilde{L}_{t+s}$ be the corresponding sequence of leader masses. We will use $\hat{L}_{t+s}$ to denote the sequences with the policy choice $a_{t+s}=e, \forall s$. Note that since $L_{t} \geq L^{*}>\bar{L}_{e}, \hat{L}_{t+s} \leq \tilde{L}_{t+s}$ holds, with strict inequality if the sequence contains at least one $b$ before $t+s-1$. (This can be shown from $L_{t+1}=L_{t} \gamma_{a}+\bar{L}_{a}\left(1-\gamma_{a}\right), \gamma_{b}>\gamma_{e}$, and $\left.\bar{L}_{b}>\bar{L}_{e}.\right)$

The total surplus from the sequence of policy choices $\tilde{a}_{s}$ is then given by:

$$
\mathbf{S}\left(\tilde{a}_{t}, \tilde{a}_{t+1}, \ldots, \tilde{a}_{t+T-1}\right) \equiv \sum_{s=0}^{T-1} \beta^{s}\left[\left(1-\delta_{\tilde{a}_{s}}\right) \tilde{L}_{t+s} \pi_{\ell}+\lambda_{\tilde{a}_{s}}\left(1-\tilde{L}_{t+s}\right) \pi_{f}^{\prime}\right]+\beta^{T} S\left(\tilde{L}_{s+T}\right) .
$$

Therefore, $\mathbf{S}\left(\tilde{a}_{t}, \tilde{a}_{t+1}, \ldots, \tilde{a}_{t+T-1}\right)$ is linear in $\pi_{f}^{\prime}$ and

$$
\frac{\partial}{\partial \pi_{f}^{\prime}} \mathbf{S}\left(\tilde{a}_{t}, \tilde{a}_{t+1}, \ldots, \tilde{a}_{t+T-1}\right)=\sum_{s=0}^{T-1} \beta^{s} \lambda_{\tilde{a}_{s}}\left(1-\tilde{L}_{t+s}\right)
$$


Thus

$$
\frac{\partial}{\partial \pi_{f}^{\prime}} \mathbf{S}(e, e, \ldots, e)-\frac{\partial}{\partial \pi_{f}^{\prime}} \mathbf{S}\left(\tilde{a}_{t}, \tilde{a}_{t+1}, \ldots, \tilde{a}_{t+T-1}\right)=\sum_{s=0}^{T-1} \beta^{s}\left[\lambda_{e}\left(1-\hat{L}_{t+s}\right)-\lambda_{\tilde{a}_{s}}\left(1-\tilde{L}_{t+s}\right)\right] .
$$

Let $B$ be the set of all sequences of policy choices $\left\{\tilde{a}_{t+s}\right\}_{s=0}^{T-1}$ such that $\tilde{a}_{t+s}=b$ for some $s$. Let $\left(\tilde{a}_{t}, \ldots, \tilde{a}_{t+T-1}\right) \in B$ and let $\left(\tilde{L}_{t}, \ldots, \tilde{L}_{t+T-1}\right)$ be the corresponding sequence of leader masses. Since $\lambda_{e} \geq \lambda_{\tilde{a}_{s}}$ and $1-\hat{L}_{t+s} \geq 1-\tilde{L}_{t+s}$ with at least one strict inequality (and $\hat{L}_{t+s}, \tilde{L}_{t+s}<1$ for $s \geq 1$ ), it follows that

$$
\frac{\partial}{\partial \pi_{f}^{\prime}} \mathbf{S}(e, e, \ldots, e)>\frac{\partial}{\partial \pi_{f}^{\prime}} \mathbf{S}\left(\tilde{a}_{t}, \tilde{a}_{t+1}, \ldots, \tilde{a}_{t+T-1}\right) .
$$

Moreover, since the set $B$ is finite,

$$
\frac{\partial}{\partial \pi_{f}^{\prime}} \mathbf{S}(e, e, \ldots, e)>\max _{\left(\tilde{a}_{t}, \ldots, \tilde{a}_{t+T-1}\right) \in B} \frac{\partial}{\partial \pi_{f}^{\prime}} \mathbf{S}\left(\tilde{a}_{t}, \tilde{a}_{t+1}, \ldots, \tilde{a}_{t+T-1}\right) .
$$

Thus for $\pi_{f}^{\prime}$ sufficiently large, $\mathbf{S}(e, e, \ldots, e)>\mathbf{S}\left(\tilde{a}_{t}, \tilde{a}_{t+1}, \ldots, \tilde{a}_{t+T-1}\right)$ for all possible sequences $\tilde{a}_{t+s}$. Therefore $a_{t+s}=e$ is chosen for all $s<T$.

For $s \geq T$, by the choice of $T, L_{t+T}<L^{*}$ holds. Thus $a_{t+s}=e$ for all $s \geq T$ as well. 


\section{References}

[1] Acemoglu, Daron and James A. Robinson (2012). Why Nations Fail, New York, Crown Publishers.

[2] Aghion, Philippe, and Peter Howitt (1992). "A Model of Growth through Creative Destruction," Econometrica 60, 323-351.

[3] Barseghyan, Levon and Riccardo DiCecio (2011). "Entry Costs, Industry Structure, and Cross-Country Income and TFP Differences," Journal of Economic Theory 146, 18281851.

[4] Bellettini, Giorgio and Gianmarco I. P. Ottaviano (2005). "Special Interests and Technological Change," Review of Economic Studies 72, 43-56.

[5] Bergemann, Dirk and Juuso Välimäki (2003). "Dynamic Common Agency," Journal of Economic Theory 111, 23-48.

[6] Bernheim, B. Douglas and Michael D. Whinston (1986). "Menu Auctions, Resource Allocation, and Economic Influence," Quarterly Journal of Economics 101, 1-31.

[7] Bridgman, Benjamin R.; Igor D. Livshits; and James C. MacGee (2004). "For Sale: Barriers to Riches," mimeo. Louisiana State University and University of Western Ontario.

[8] De Soto, Hernando (1989). The Other Path, New York: Basic Books.

[9] De Soto, Hernando (2000). The Mystery of Capital, New York: Basic Books.

[10] Djankov, Simeon (2009). "The Regulation of Entry: A Survey," World Bank Research Observer 24, 183-203.

[11] Djankov, Simeon; Rafael La Porta; Florencio Lopez-de-Silanes; and Andrei Shleifer (2002). "The Regulation of Entry," Quarterly Journal of Economics 117, 1-37.

[12] Foster, Lucia; John Haltiwanger; and C. J. Krizan (2001). "Aggregate Productivity Growth: Lessons from Microeconomic Evidence," in Charles R. Hulten, Edwin R. Dean, 
and Michael J. Harper (eds.) New Developments in Productivity Analysis, Chicago: University of Chicago Press.

[13] Foster, Lucia; John Haltiwanger; and C. J. Krizan (2006). "Market Selection, Reallocation, and Restructuring in the U.S. Retail Trade Sector in the 1990s," Review of Economics and Statistics 88, 748-758.

[14] Grossman, Gene M. and Elhanan Helpman (1991). Innovation and Growth in the Global Economy, Cambridge: MIT Press.

[15] Grossman, Gene M. and Elhanan Helpman (1994). "Protection for Sale," American Economic Review 84, 833-850.

[16] Hayek, Friedrich A. (1979). Law, Legislation, and Liberty; Volume 3: The Political Order of a Free People, Chicago: University of Chicago Press.

[17] Krusell, Per and José-Víctor Ríos-Rull (1996). "Vested Interests in a Positive Theory of Stagnation and Growth," Review of Economic Studies 63, 301-329.

[18] Moscoso Boedo, Hernan J. and Toshihiko Mukoyama (2012). "Evaluating the Effects of Entry Regulations and Firing Costs on International Income Differences," Journal of Economic Growth 17, 143-170.

[19] North, Douglass C. (1990). Institutions, Institutional Change and Economic Performance, Cambridge: Cambridge University Press.

[20] Olson, Mancur (1965). The Logic of Collective Action: Public Goods and the Theory of Groups, Cambridge: Harvard University Press.

[21] Olson, Mancur (1982). The Rise and Decline of Nations, New Haven: Yale University Press.

[22] Parente, Stephen and Edward C. Prescott (1999). "Monopoly Rights: A Barrier to Riches," American Economic Review 89, 1216-1233. 
[23] Rockafellar, R. Tyrrell (1970). Convex Analysis, Princeton: Princeton University Press.

[24] Stokey, Nancy L. and Robert E. Lucas, Jr. with Edward C. Prescott (1989). Recursive Methods in Economic Dynamics, Cambridge: Harvard University Press. 